\title{
Adjoint functors between categories of Hilbert $C^{*}$-modules
}

\author{
Pierre Clare $\quad$ Tyrone Crisp ${ }^{*} \quad$ Nigel Higson ${ }^{\dagger}$
}

\begin{abstract}
Let $E$ be a (right) Hilbert module over a $C^{*}$-algebra $A$. If $E$ is equipped with a left action of a second $C^{*}$-algebra $B$, then tensor product with $E$ gives rise to a functor from the category of Hilbert $B$-modules to the category of Hilbert $A$-modules. The purpose of this paper is to study adjunctions between functors of this sort. We shall introduce a new kind of adjunction relation, called a local adjunction, that is weaker than the standard concept from category theory. We shall give several examples, the most important of which is the functor of parabolic induction in the tempered representation theory of real reductive groups. Each local adjunction gives rise to an ordinary adjunction of functors between categories of Hilbert space representations. In this way we shall show that the parabolic induction functor has a simultaneous left and right adjoint, namely the parabolic restriction functor constructed in [CCH16].
\end{abstract}

Keywords: Hilbert $C^{*}$-modules; adjoint functors; parabolic induction MSC2010: 46L08, 18A40

\section{Introduction}

Let $A$ be a $C^{*}$-algebra and denote by ${ }_{A} \mathcal{H}$ the category of non-degenerate Hilbert space representations of $A$. This is obviously a category of interest

*Partially supported by the Danish National Research Foundation through the Centre for Symmetry and Deformation (DNRF92).

${ }^{\dagger}$ Partially supported by the US National Science Foundation DMS-1101382. 
when $A$ is a group $C^{*}$-algebra, since it is isomorphic to the category of unitary representations of the group. Similarly, if $A$ is the reduced $C^{*}$-algebra of a real reductive group, then ${ }_{A} \mathcal{H}$ is isomorphic to the category of tempered unitary representations of the group.

In addition, denote by $\mathcal{H}_{A}$ the category of right Hilbert $A$-modules (a Hilbert module is a Banach module whose norm is obtained from an associated $A$-valued inner product; see [BLM04], [Lan95], or Section 2 for a quick review). The role of this category in representation theory is a bit less clear, but for example if $A$ is the reduced $C^{*}$-algebra of a real reductive group $G$, then each discrete series representation of $G$ determines an object in $\mathcal{H}_{A}$. More generally, the category $\mathcal{H}_{A}$ captures the topology of the dual space $\widehat{A}$ of irreducible representations of $A$ (this is the tempered dual in our reduced group $C^{*}$-algebra example). In contrast, the category ${ }_{A} \mathcal{H}$ of Hilbert space representations is more closely related to the structure of the dual as a set or measurable space.

In this paper we shall study the categories ${ }_{A} \mathcal{H}$ and $\mathcal{H}_{A}$ with a particular view to the case of the reduced $C^{*}$-algebra of a real reductive Lie group $G$. We shall further develop the analysis of the parabolic induction functor

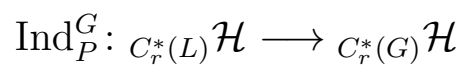

from the perspective of Hilbert modules that was begun in Cla13 and [CCH16]. Here $L$ is a Levi subgroup of a parabolic subgroup $P \subseteq G$. We shall examine the relationship between parabolic induction and the functor of parabolic restriction

$$
\operatorname{Res}_{P}^{G}:{ }_{C_{r}^{*}(G)} \mathcal{H} \longrightarrow C_{r}^{*}(L) \mathcal{H}
$$

that we introduced in $\mathrm{CCH} 16$. We shall prove that parabolic induction and restriction are left and right adjoints of one another. In fact we shall prove this as a consequence of a stronger statement involving the Hilbert module categories $\mathcal{H}_{C_{r}^{*}(G)}$ and $\mathcal{H}_{C_{r}^{*}(L)}$. See Theorems 5.1 and 5.2 .

If $A$ and $B$ are $C^{*}$-algebras, then by a correspondence from $A$ to $B$ we shall mean a Hilbert $B$-module equipped with a nondegenerate representation of $A$ as adjointable Hilbert $B$-module endomorphisms. Associated to such a correspondence $F$ there are functors

$$
{ }_{B} \mathcal{H} \longrightarrow{ }_{A} \mathcal{H} \text { and } \mathcal{H}_{A} \longrightarrow \mathcal{H}_{B}
$$


that are constructed using Hilbert module tensor products. The functors of parabolic induction and restriction from [Cla13 and [CCH16] are of this type.

In the algebraic setting, where $A$ and $B$ are rings with unit, it is well known that if $F$ is an $A$-B-bimodule, then the associated tensor product functors

$$
{ }_{B} \operatorname{Mod} \longrightarrow{ }_{A} \operatorname{Mod} \text { and } \operatorname{Mod}_{A} \longrightarrow \operatorname{Mod}_{B}
$$

between left and right module categories always admit right adjoints, namely $X \mapsto \operatorname{Hom}_{A}(F, X)$ and $Y \mapsto \operatorname{Hom}_{B}(F, Y)$ respectively. But in the $C^{*}$-setting the extra symmetry imposed by the $*$-operation means that every right adjoint is also a left adjoint, and the existence of an adjoint functor in these circumstances is a much more delicate matter.

Kajiwara, Pinzari and Watatani [KPW04] have obtained necessary and sufficient conditions on a correspondence $F$ for the tensor-product functor between Hilbert module categories to admit an adjoint. Unfortunately their conditions, which we shall review in Section 2.4, immediately rule out many naturally-occurring examples of correspondences. Most notably from our point of view, the conditions are not satisfied by the correspondences associated to parabolic induction and restriction. Comparing with the theory of smooth representations of $p$-adic reductive groups-where, as Bernstein showed ([Ber87], cf. [Ren10]), the parabolic-induction functor admits both left and right adjoints, and where those adjoints are close to being identicalwe were led to look for a weaker notion of adjunction for Hilbert modules that would apply in particular to parabolic induction. The main novelty of this paper is to define such a notion, and to study some of its properties.

If $F$ is a correspondence from $A$ to $B$, and $E$ is a correspondence from $B$ to $A$, we shall say that the associated tensor-product functors

$$
\mathcal{H}_{A} \stackrel{\otimes_{A} F}{\longrightarrow} \mathcal{H}_{B} \quad \text { and } \quad \mathcal{H}_{B} \stackrel{\otimes_{B} E}{\longrightarrow} \mathcal{H}_{A}
$$

are locally adjoint if there are natural isomorphisms

$$
\mathfrak{K}_{B}\left(X \otimes_{A} F, Y\right) \stackrel{\cong}{\longrightarrow} \mathfrak{K}_{A}\left(X, Y \otimes_{B} E\right)
$$

between spaces of compact Hilbert module operators. See Definition 3.1 for details. Not every morphism between Hilbert modules is compact, so our definition is not the usual definition of an adjunction. Nor is it obtained from the usual definition by adjusting to take into account the $C^{*}$-structure 
[GLR85 on the categories $\mathcal{H}_{A}$ and $\mathcal{H}_{B}$. But if two tensor product functors are adjoint in the usual sense, then the natural isomorphisms coming from the adjunction restrict to isomorphisms between spaces of compact operators, as above, and so adjunction implies local adjunction. See Corollary 3.9. The converse is rarely true: in general local adjunction is a genuinely weaker condition.

The commutative case illustrates the distinction between adjoint and local adjoint quite well. If $Y$ is a quotient of a compact Hausdorff space $X$, then the pullback functor $\mathcal{H}_{C(Y)} \rightarrow \mathcal{H}_{C(X)}$ has an adjoint if and only if $X$ is a finite cover of $Y$, while it has a local adjoint if and only if $X$ is a finite branched cover. See Section 4.1. In addition, if $U$ is an open subset of $X$, then the pushforward $\mathcal{H}_{C_{0}(U)} \rightarrow \mathcal{H}_{C(X)}$ always has a local adjoint, but it has an adjoint if and only if $U$ is both open and closed in $X$.

In Sections 3.3 and 3.7 we characterize locally adjoint pairs of tensor product functors, and show that the condition of local adjointability is equivalent to the condition of "finite numerical index" considered in [KPW04]. Returning to categories of Hilbert space representations, we show that if two tensor product functors are locally adjoint, then the associated functors between the Hilbert space representation categories ${ }_{A} \mathcal{H}$ and ${ }_{B} \mathcal{H}$ are (two-sided) adjoints in the usual sense. See Theorem 3.15.

On the basis of all this it is easy to analyze several examples, and we shall do so in Section 4. Our main example of parabolic induction is considered in Section 5. As a particular consequence we show that the parabolic restriction functor constructed in [CCH16] is a two-sided adjoint to the parabolic induction functor from tempered unitary representations of a Levi factor of a reductive group $G$ to tempered unitary representations of $G$. This adjoint functor appears to be new.

\section{Background and Notation}

\subsection{Hilbert Modules and Correspondences}

Let $A$ be a $C^{*}$-algebra. Recall that a Hilbert $A$-module is a right $A$-module $X$ that is equipped with an inner product

$$
\left\langle{ }_{-},{ }_{-}\right\rangle: X \times X \longrightarrow A
$$


that is $\mathbb{C}$-antilinear in the first variable, $\mathbb{C}$-linear in the second variable, and satisfies

$$
\langle x, y a\rangle=\langle x, y\rangle a, \quad\langle x, y\rangle^{*}=\langle y, x\rangle \quad \text { and } \quad\langle x, x\rangle \geq 0
$$

for all $x, y \in X$ and $a \in A$. In addition the formula

$$
\|x\|_{X}^{2}:=\|\langle x, x\rangle\|_{A}
$$

is required to define a complete norm on $X$. See for example BLM04, Chapter 8] or [Lan95] for an introduction to this concept. (But note that the name "Hilbert $C^{*}$-module" is used in BLM04 to refer to what we are calling Hilbert module, while the name "Hilbert module" is used in [BLM04] to refer to a different concept.)

A map $T: X \rightarrow Y$ between Hilbert $A$-modules is adjointable if there is a map $T^{*}: Y \rightarrow X$ (necessarily unique) satisfying

$$
\langle T x, y\rangle=\left\langle x, T^{*} y\right\rangle
$$

for all $x \in X$ and $y \in Y$. Adjointable operators are automatically $A$-linear and bounded, but the converse is not true, in general. We shall denote by $\mathfrak{B}_{A}(X, Y)$ the Banach space of all adjointable operators from $X$ to $Y$. The space $\mathfrak{B}_{A}(X, X)$ of adjointable operators from $X$ to itself is a $C^{*}$-algebra in the operator norm.

Definition 2.1. Let $B$ be a second $C^{*}$-algebra and let $F$ be a Hilbert $B$ module. A $*$-homomorphism $A \rightarrow \mathfrak{B}_{B}(F, F)$ is nondegenerate if the elements af $(a \in A, f \in F)$ span a dense subspace of $F$.

Definition 2.2. By a correspondence from $A$ to $B$ we shall mean a Hilbert $B$-module $F$ equipped with a nondegenerate $*$-homomorphism from $A$ into $\mathfrak{B}_{B}(F, F)$.

Remarks 2.3. A correspondence from $A$ to $B$ is in particular an $A$ - $B$ bimodule, but the definition is asymmetric in that no $A$-valued inner product is implied. The condition of nondegeneracy will not play a crucial role in what follows. Nevertheless we shall assume it since it in any case holds in the main examples of interest to us. 


\subsection{Compact Operators}

If $X$ and $Y$ are Hilbert $A$-modules, and if $x \in X$ and $y \in Y$, then the formula

$$
y \otimes x^{*}: z \mapsto y\langle x, z\rangle
$$

defines an adjointable operator from $X$ to $Y$; the adjoint is $x \otimes y^{*}$. The operator-norm closure of the linear span of the operators

$$
y \otimes x^{*} \in \mathfrak{B}_{A}(X, Y)
$$

is by definition the subspace of $A$-compact operators, denoted $\mathfrak{K}_{A}(X, Y)$. The composition, on either side, of a compact operator with an adjointable operator is compact.

To add some algebraic substance to the notation used in (2.4) and (2.5), we introduce the following concept:

Definition 2.6. Let $X$ be a Hilbert $A$-module. The conjugate of $X$, denoted $X^{*}$, is the complex conjugate of the vector space $X$, equipped with left $A$ module structure defined by

$$
a \cdot x^{*}=\left(x a^{*}\right)^{*}
$$

Here $x^{*} \in X^{*}$ denotes the image of $x \in X$ under the obvious conjugate $\mathbb{C}$-linear isomorphism $X \rightarrow X^{*}$.

Remark 2.7. The term adjoint would usually be a more appropriate name for $X^{*}$ than conjugate, but the word will be used quite heavily in other senses in this paper.

Using the left $A$-module structure on $X^{*}$ we find that

$$
y \cdot a \otimes x^{*}=y \otimes a \cdot x^{*} \in \mathfrak{B}_{A}(X, Y),
$$

and so the formula $(2.4)$ defines a $\mathbb{C}$-linear map

$$
Y \otimes_{A}^{\mathrm{alg}} X^{*} \longrightarrow \mathfrak{B}_{A}(X, Y) .
$$

The compact operators therefore constitute a completion of the algebraic tensor product $Y \otimes_{A}^{\text {alg }} X^{*}$ within $\mathfrak{B}_{A}(X, Y)$. We shall return to this perspective on compact operators in a little while. 
We shall need one more idea about compact operators. Let $X$ be a Hilbert $A$-module. The space $\mathfrak{K}_{A}(A, X)$ of compact adjointable operators from the Hilbert module $A$ to $X$ is itself a Hilbert $A$-module under the inner product

$$
\langle S, T\rangle=S^{*} T \text {. }
$$

Here the operator $S^{*} T: A \rightarrow A$ is left multiplication by some unique element of $A$, and we identify $S^{*} T$ with that element so as to obtain an $A$-valued inner product. Each operator in $\mathfrak{K}_{A}(A, X)$ is of the form

$$
T: a \mapsto x a
$$

for some unique element $x \in X$. Moreover all these operators are adjointable, with adjoint

$$
T^{*}: y \mapsto\langle x, y\rangle .
$$

Finally, $T$ and $T^{*}$ are compact, as can be seen using an approximate identity in $A$. We arrive at the following results (see also [BLM04, Proposition 8.1.11]).

Lemma 2.9. The Hilbert $A$-module $X$ is isomorphic to the Hilbert A-module $\mathfrak{K}_{A}(A, X)$ via the map that associates to $x \in X$ the compact operator $a \mapsto$ $x a$.

Lemma 2.10. The conjugate $X^{*}$ of a Hilbert $A$-module $X$ is isomorphic to the $A$-module $\mathfrak{K}_{A}(X, A)$ via the map that associates to $x^{*} \in X^{*}$ the compact operator $y \mapsto\langle x, y\rangle$.

\subsection{Functors on Hilbert Modules}

Definition 2.11. If $A$ is a $C^{*}$-algebra, then denote by $\mathcal{H}_{A}$ the category whose objects are right Hilbert $A$-modules and whose morphisms are adjointable maps between Hilbert modules.

Within the algebraic context, bimodules give rise to functors between module categories via tensor product. This is so in the Hilbert module context, too, thanks to the following construction.

Definition 2.12. Let $X$ be a Hilbert $A$-module and let $F$ be a correspondence from $A$ to $B$. The (internal) tensor product $X \otimes_{A} F$, which is a Hilbert $B$-module, is the completion of the algebraic tensor product $X \otimes_{A}^{\text {alg }} F$ in the norm induced by the $B$-valued inner product

$$
\left\langle x_{1} \otimes f_{1}, x_{2} \otimes f_{2}\right\rangle_{X \otimes_{A} F}:=\left\langle f_{1},\left\langle x_{1}, x_{2}\right\rangle_{X} \cdot f_{2}\right\rangle_{F} .
$$


See [Lan95, Chapter 4] for basic information on the internal tensor product construction. One has natural isomorphisms

$$
A \otimes_{A} F \stackrel{\cong}{\longrightarrow} F \quad \text { and } \quad F \otimes_{B} B \stackrel{\cong}{\longrightarrow} F
$$

via multiplication.

If $F$ is a correspondence from $A$ to $B$, then internal tensor product with $F$ gives rise to a tensor product functor

$$
F: \mathcal{H}_{A} \longrightarrow \mathcal{H}_{B}
$$

(as indicated, we shall use the same letter for the bimodule and the functor), since the tensor product of an adjointable operator with the identity operator on $F$ is an adjointable operator between tensor product modules.

It is interesting to note that subject to a natural continuity condition and compatibility with the adjoint operation, every functor between Hilbert module categories is a tensor product functor. We shall not use this fact, but here is a short summary.

Definition 2.13. A functor $F$ between categories of Hilbert modules is called a *-functor if it is $\mathbb{C}$-linear on morphisms, and satisfies $F\left(T^{*}\right)=F(T)^{*}$ for every adjointable operator $T$.

Definition 2.14. A $*$-functor $F: \mathcal{H}_{A} \rightarrow \mathcal{H}_{B}$ is strongly continuous if for every object $X \in \mathcal{H}_{A}$, the $*$-homomorphism

$$
F: \mathfrak{K}_{A}(X, X) \rightarrow \mathfrak{B}_{B}(F(X), F(X))
$$

is nondegenerate.

Theorem 2.15. [Ble97, Theorem 5.3] The category of strongly continuous *-functors $\mathcal{H}_{A} \rightarrow \mathcal{H}_{B}$ (and natural transformations) is equivalent to the category of correspondences from $A$ to $B$ (and adjointable operators compatible with the $A-B$ bimodule structure).

The equivalence is given in one direction by sending a functor $F$ to the correspondence $F(A)$, and in the other direction by sending a correspondence $F$ to the associated tensor product functor. 
Remark 2.16. In addition a strongly continuous *-functor $\mathcal{H}_{A} \rightarrow \mathcal{H}_{B}$ is an equivalence (with inverse a strongly continuous $*$-functor) if and only if the associated correspondence is a Morita equivalence between $A$ and $B$ : see [BLM04, 8.1.2, 8.2.20]. If $A$ and $B$ are Morita equivalent, then the dual spaces $\widehat{A}$ and $\widehat{B}$ are homeomorphic (moreover the converse also holds if $A$ and $B$ are commutative). So we see that $\mathcal{H}_{A}$ carries information about the structure of $\widehat{A}$ as a topological space, as remarked in the introduction.

\subsection{Adjunctions}

We shall start with the standard definition from category theory.

Definition 2.17. Let $A$ and $B$ be $C^{*}$-algebras, and let $E$ and $F$ be correspondences from $B$ to $A$ and from $A$ to $B$, respectively, determining tensor product functors

$$
\mathcal{H}_{A} \stackrel{\otimes_{A} F}{\longrightarrow} \mathcal{H}_{B} \quad \text { and } \quad \mathcal{H}_{B} \stackrel{\otimes_{B} E}{\longrightarrow} \mathcal{H}_{A} .
$$

An adjunction between $F$ and $E$ is a natural isomorphism

$$
\Phi_{X, Y}: \mathfrak{B}_{B}\left(X \otimes_{A} F, Y\right) \stackrel{\cong}{\longrightarrow} \mathfrak{B}_{A}\left(X, Y \otimes_{B} E\right),
$$

or in other words a natural equivalence between the left and right sides in (2.18), considered as functors from the product category $\mathcal{H}_{A}^{\mathrm{op}} \times \mathcal{H}_{B}$ to the category of sets.

But unlike the ordinary situation in category theory, there is no real distinction between left adjoints and right adjoints in the context of Hilbert modules: given an adjunction $\Phi_{X, Y}$ as above, the formula

$$
\Phi_{X, Y}^{*}: T \mapsto\left(\Phi_{X, Y}\right)^{-1}\left(T^{*}\right)^{*}
$$

defines an adjunction

$$
\Phi_{X, Y}^{*}: \mathfrak{B}_{A}\left(Y \otimes_{B} E, X\right) \stackrel{\cong}{\longrightarrow} \mathfrak{B}_{B}\left(Y, X \otimes_{A} F\right)
$$

that reverses the role of $E$ and $F$ in Definition 2.17 .

Definition 2.20. Let $\Phi$ be an adjunction, as in Definition 2.17. 
(a) A unit for $\Phi$ is a bounded, adjointable $A$-bimodule map

$$
\eta: A \longrightarrow F \otimes_{B} E
$$

that defines $\Phi_{X, Y}$ by means of the commuting diagram

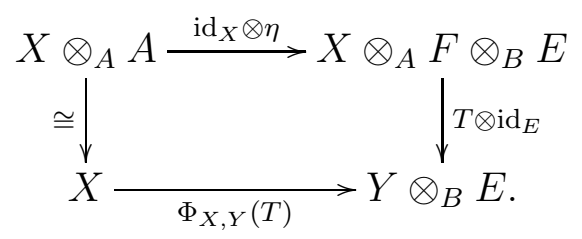

(b) A counit for $\Phi$ is a bounded, adjointable $B$-bimodule map

$$
\varepsilon: E \otimes_{A} F \longrightarrow B
$$

that defines the inverse of the isomorphism $\Phi_{X, Y}$ in (2.18) by means of the commuting diagram

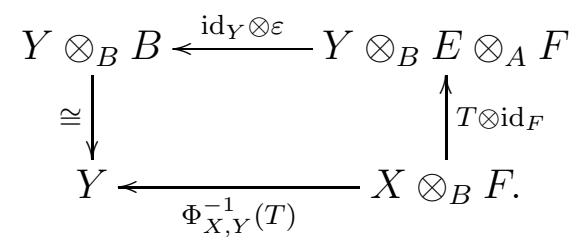

Proposition 2.21. Every adjunction admits a unique unit and counit.

Proof. This is standard. For instance the unit is the image of the identity operator on $F$ under the map

$$
\Phi_{A, F}: \mathfrak{B}_{B}(F, F) \stackrel{\cong}{\longrightarrow} \mathfrak{B}_{A}\left(A, F \otimes_{B} E\right),
$$

See [ML98, IV.1], for example.

No continuity conditions are imposed on the isomorphisms $\Phi_{X, Y}$ in an adjunction. But in fact continuity is automatic, as the following calculation shows.

Lemma 2.22. Let $\Phi$ be an adjunction, as in Definition 2.17. Each map

$$
\Phi_{X, Y}: \mathfrak{B}_{B}\left(X \otimes_{A} F, Y\right) \stackrel{\cong}{\longrightarrow} \mathfrak{B}_{A}\left(X, Y \otimes_{B} E\right),
$$

is a linear, topological isomorphism. 
Proof of the Lemma. It is clear from the definition of unit that $\Phi_{X, Y}$ is linear, with norm bounded by the operator norm of the unit map from $A$ into $F \otimes_{B} E$. The inverse is likewise linear, with norm bounded by the norm of the counit.

The following definition very slightly elaborates on Definition 2.6 .

Definition 2.23. Let $A$ and $B$ be $C^{*}$-algebras and let $F$ be a correspondence from $A$ to $B$. The conjugate of $F$ is the Hilbert module conjugate $F^{*}$, as in Definition 2.6, equipped with right $A$-module structure defined by

$$
f^{*} \cdot a=\left(a^{*} f\right)^{*}
$$

The conjugate $F^{*}$ of a correspondence from $A$ to $B$ is a $B$ - $A$-bimodule, but, as it stands, is not a correspondence from $B$ to $A$ : there is no obvious $A$-valued inner product. Kajiwara, Pinzari and Watatani [KPW04] relate the existence of such an inner product on $F^{*}$ to the existence of adjoint functors, as follows.

Theorem 2.24. [KPW04, Theorem 4.4(2), Theorem 4.13] A tensor product functor from $\mathcal{H}_{A}$ to $\mathcal{H}_{B}$, induced from a correspondence $F$, has an adjoint tensor product functor if and only if all the following conditions are met:

(a) The conjugate $B$-A-bimodule $F^{*}$ carries an A-valued inner product making it into a correspondence from $B$ to $A$.

(b) The conjugate operator space structure on $F^{*}$ is completely boundedly equivalent to the Hilbert $A$-module operator space structure on $F^{*}$.

(c) The left action of $A$ on $F$ is through a *-homomorphism from $A$ into $\mathfrak{K}_{B}(F, F)$.

(d) The left action of $B$ on $F^{*}$ is through a*-homomorphism from $B$ into $\mathfrak{K}_{A}\left(F^{*}, F^{*}\right)$.

When these conditions are met, the adjoint functor from $\mathcal{H}_{B}$ to $\mathcal{H}_{A}$ is given by tensor product with the correspondence $F^{*}$.

Condition (b) will be explained in the next section, where we shall also give a proof of Theorem 2.24 in the course of our study of the weaker notion of local adjunction. The full relationship of our results to those of [KPW04] will be discussed in detail in Section 3.7. 
Example 2.25. Consider the case of unital $C^{*}$-algebras $A$ and $B$. If $F$ is a correspondence satisfying condition (a) of Theorem 2.24 , then the conditions (c) and (d) are equivalent to requiring that $F$ be finitely generated (and hence projective, cf. [BLM04, Theorem 8.1.27]) as a module over $B$ and $A$, respectively, while the analytic condition (b) follows automatically from (c) and (d): see [KPW04, Example 2.31]. (We note that in the non-unital setting, the conditions (b), (c) and (d) are independent of one another: see the examples in Sections 4.5.3 and 4.5.1.) Combined with a theorem of Morita on adjunctions in the algebraic setting [Mor65, Theorem 4.1], we find that the following are equivalent for unital $C^{*}$-algebras $A$ and $B$ :

(a) The tensor product functor $\otimes_{A} F: \mathcal{H}_{A} \rightarrow \mathcal{H}_{B}$ has an adjoint.

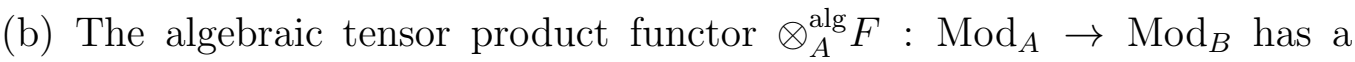
two-sided adjoint.

This situation is reminiscent of Beer's result, that two unital $C^{*}$-algebras are Morita equivalent as $C^{*}$-algebras if and only they are Morita equivalent as rings [Bee82, Theorem 1.8].

\subsection{Hilbert Modules as Operator Spaces}

Recall that an operator space structure on a vector space $X$ is a sequence of Banach space norms on the spaces $M_{n}(X)$ of $n \times n$ matrices with entries from $X$ such that

(a) the norm of a block diagonal matrix is the largest of the norms of its diagonal blocks; and

(b) the norm of a three-fold product $A B C$, where $A$ and $C$ are scalar $n \times n$ matrices, and $B$ is an $n \times n$ matrix with entries from $X$, is no more than the product of the norms of the matrices $A, B$ and $C$ (we use the operator norm for the scalar matrices).

See [ER00] or [BLM04]. An operator space is of course a vector space with an operator space structure.

Example 2.26. The above conditions hold when $X$ is a closed subspace of a $C^{*}$-algebra $B$ and the norms on $M_{n}(X)$ are inherited from the $C^{*}$-algebra norm on $M_{n}(B)$. 

if

A linear map $T: X \rightarrow Y$ between operator spaces is completely bounded

$$
\|T\|_{\mathrm{cb}}=\sup _{n}\left\|M_{n}(T)\right\|<\infty,
$$

where $M_{n}(T): M_{n}(X) \rightarrow M_{n}(Y)$ is defined by applying $T$ entrywise. An isomorphism of operator spaces will mean, for us, a linear isomorphism $T$ : $X \rightarrow Y$ such that both $T$ and $T^{-1}$ are completely bounded. We shall use the term complete isometric isomorphism when

$$
\|T\|_{\mathrm{cb}}=\left\|T^{-1}\right\|_{\mathrm{cb}}=1 .
$$

Example 2.27. Every operator space is completely isometrically isomorphic to a closed subspace of a $C^{*}$-algebra. See for example [ER00, Chapter 2] for an exposition.

Suppose now that $X$ is a right Hilbert $A$-module. Then $M_{n}(X)$ is a Hilbert $M_{n}(A)$-module in a natural way, namely the right action of $M_{n}(A)$ on $M_{n}(X)$ is given by matrix multiplication, and the $M_{n}(A)$-valued inner product is

$$
\langle S, T\rangle_{i, j}=\sum_{k=1}^{n}\left\langle S_{k j}, T_{k j}\right\rangle .
$$

This observation gives $X$ an operator space structure. See [BLM04, 8.2.1].

Example 2.28. If $A$ is a $C^{*}$-algebra, then the operator space norms on $M_{n}(A)$ induced by the inner product $\langle a, b\rangle=a^{*} b$ on $A$ are the canonical $C^{*}$-algebra norms.

Example 2.29. If $X$ is a closed subspace of $\mathfrak{B}_{A}(Y, Z)$, where $Y$ and $Z$ are Hilbert $A$-modules, then the operator space structure on $X$ associated to the $\mathfrak{B}_{A}(Y)$-valued inner product $\langle S, T\rangle=S^{*} T$ coincides with the one given by the natural embedding of $\mathfrak{B}_{A}(Y, Z)$ as a corner of the $C^{*}$-algebra $\mathfrak{B}_{A}(Y \oplus Z)$.

For instance, if $X$ is a Hilbert $A$-module then the isomorphism $X \cong$ $\mathfrak{K}_{A}(A, X)$ of Lemma 2.9 is completely isometric for the operator space structure induced on $X$ by the $A$-valued inner product, and the operator space structure on $\mathfrak{K}_{A}(A, X)$ induced by its embedding into $\mathfrak{K}_{A}(A \oplus X)$.

Example 2.30. If $H$ is a Hilbert space, then the operator space structure associated to the inner product on $H$ is the column Hilbert space structure [BLM04, 1.2.23] coming from the identification $H \cong \mathfrak{B}(\mathbb{C}, H)$. 
The assignment of an operator space structure to each Hilbert module, as above, gives a functorial embedding of the category of Hilbert modules and adjointable maps into the category of operator spaces and completely bounded maps:

Theorem 2.31. [BLM04, Proposition 8.2.2] If $X$ and $Y$ are right Hilbert A-modules, then every bounded A-linear map $X \rightarrow Y$ is completely bounded.

We are going to use operator spaces to treat Hilbert $A$-modules $X$ and their conjugate modules $X^{*}$ introduced in Definition 2.6 on a somewhat equal footing. To this end, we recall the following concept:

Definition 2.32. If $X$ is any operator space, then its conjugate $X^{*}$ is the complex conjugate vector space, equipped with the norms

$$
\left\|\left[x_{i j}\right]\right\|_{M_{n}\left(X^{*}\right)}=\left\|\left[x_{j i}\right]\right\|_{M_{n}(X)},
$$

which endow it with the structure of an operator space.

Remark 2.33. The operator space $X^{*}$ is usually called the adjoint of $X$, but once again we shall try to avoid over-using this word in this paper. However we warn the reader that the term conjugate as it is used in [KPW04] refers to adjoint functors.

Example 2.34. In the case of a Hilbert $A$-module $X$, the operator space structure on $X^{*}$ provided by Definition 2.32 is the operator space structure we would obtain by viewing $X^{*}$ as a left Hilbert $A$-module (a concept that we are avoiding in this paper). See [BLM04, 1.2.25 and 8.2.3(2)].

Example 2.35. Let $H$ be a complex Hilbert space, equipped with its column operator space structure. The conjugate operator space $H^{*}$ is, as a vector space, the same as the complex conjugate Hilbert space $\bar{H}$, and the conjugate operator space structure on $H^{*}$ is the same as the row Hilbert space structure [BLM04, 1.2.23] on $\bar{H}$. Being a Hilbert space in its own right, $\bar{H}$ also carries a column Hilbert space structure. The identity map $I: \bar{H} \rightarrow \bar{H}$, considered as a map from the row operator space to the column operator space, has $\|I\|_{\mathrm{cb}}^{2}=\operatorname{dim} H$. In particular, this map is not completely bounded if $H$ is infinite-dimensional. See [ER00, p.56].

Example 2.36. Let $X$ be a Hilbert $A$-module, and view $X^{*}$ as a conjugate operator space as above. The isomorphism $X^{*} \cong \mathfrak{K}_{A}(X, A)$ of Lemma 2.10 is completely isometric, where $\mathfrak{K}_{A}(X, A)$ is viewed as a subspace of $\mathfrak{K}_{A}(X \oplus A)$ as in Example 2.29, 


\subsection{Operator Modules}

If a $C^{*}$-algebra $A$ (or more generally a Banach algebra of operators on a Hilbert space) acts on a Banach space $X$, then there are natural induced actions

$$
M_{n}(A) \times M_{n}(X) \longrightarrow M_{n}(X)
$$

that combine the given action with matrix multiplication. When $X$ is an operator space we shall always assume that these actions are completely contractive in the sense that

$$
\|a \cdot x\|_{M_{n}(X)} \leq\|a\|_{M_{n}(A)}\|x\|_{M_{n}(X)} .
$$

The same will go for right actions instead of left actions, and we shall use the term operator module to describe this situation (the term h-module is used in [BLM04, Section 3.1.3]; this is a reference to the Haagerup tensor product that we shall review below).

\subsection{The Haagerup Tensor Product}

There are several notions of tensor product for operator spaces. Here we shall need only the Haagerup tensor product, which is defined as follows. Let $A$ be a $C^{*}$-algebra, let $X$ be a right operator $A$-module, and let $Y$ be a left operator $A$-module. The Haagerup tensor product $X \otimes_{h A} Y$ is the completion of the algebraic tensor product $X \otimes_{A}^{\text {alg }} Y$ that is characterized by the following universal property: each bilinear map

$$
\Phi: X \times Y \longrightarrow Z
$$

into an operator space for which

(a) $\Phi(x a, y)=\Phi(x, a y)$, and

(b) the matrix extensions

$$
\Phi_{n}: M_{n}(X) \times M_{n}(Y) \longrightarrow M_{n}(Z)
$$

satisfy

$$
\left\|\Phi_{n}(x, y)\right\|_{M_{n}(Z)} \leq\|x\|_{M_{n}(X)}\|y\|_{M_{n}(Y)} .
$$

for all $n$ 
extends to a complete contraction from $X \otimes_{h A} Y$ to $Z$. See [BLM04, 3.4.2].

The Haagerup tensor product is associative [BLM04, Theorem 3.4.10] and functorial in both variables with respect to completely bounded module maps [BLM04, Lemma 3.4.5], and the natural isomorphism on algebraic tensor products extends to a completely isometric isomorphism

$$
\left(X \otimes_{h A} Y\right)^{*} \stackrel{\cong}{\longrightarrow} Y^{*} \otimes_{h A} X^{*} .
$$

See [BLM04, 1.5.9].

The following theorems of Blecher relate the Haagerup tensor product to the tensor product and compact operators on Hilbert modules:

Theorem 2.37. [BLM04, Theorem 8.2.11] Let $X$ be a Hilbert A-module, and let $F$ be a correspondence from $A$ to $B$. The identity map on $X \otimes_{A}^{\text {alg }} F$ extends to a completely isometric natural isomorphism

$$
X \otimes_{h A} F \stackrel{\cong}{\longrightarrow} X \otimes_{A} F
$$

from the Haagerup tensor product to the internal Hilbert module tensor product.

Theorem 2.38. BLM04, Corollary 8.2.15] Let $X$ and $Y$ be Hilbert Amodules. There is a completely isometric isomorphism of operator spaces

$$
Y \otimes_{h A} X^{*} \stackrel{\cong}{\longrightarrow} \mathfrak{K}_{A}(X, Y)
$$

mapping each elementary tensor $y \otimes x^{*}$ to the corresponding compact operator $y \otimes x^{*}$ defined in (2.5).

As in Example 2.29, $\mathfrak{K}_{A}(X, Y)$ is a closed subspace of the $C^{*}$-algebra $\mathfrak{K}_{A}(X \oplus Y)$ and it is to be viewed as an operator space in that way. Note that the operator space $Y \otimes_{h A} X^{*}$ does not depend on the inner products on $X$ and $Y$, but only on the induced operator space structures. In contrast, the action of $Y \otimes_{h A} X^{*}$ as operators $X \rightarrow Y$ appearing in Theorem 2.38 does depend on the $A$-valued inner product on $X$.

\section{Local Adjunctions for Hilbert Modules}

\subsection{Definitions and Basic Properties}

We are ready now to introduce the main concept of the paper. 
Definition 3.1. Let $A$ and $B$ be $C^{*}$-algebras, and let $E$ and $F$ be correspondences from $B$ to $A$ and from $A$ to $B$, respectively, determining tensor product functors

$$
\mathcal{H}_{A} \stackrel{\otimes_{A} F}{\longrightarrow} \mathcal{H}_{B} \quad \text { and } \quad \mathcal{H}_{B} \stackrel{\otimes_{B} E}{\longrightarrow} \mathcal{H}_{A} .
$$

A local adjunction between these functors is a natural isomorphism

$$
\Phi_{X, Y}: \mathfrak{K}_{B}\left(X \otimes_{A} F, Y\right) \stackrel{\cong}{\longrightarrow} \mathfrak{K}_{A}\left(X, Y \otimes_{B} E\right)
$$

that is, for each $X$ and $Y$, a continuous linear map.

Theorem 3.2. Let $\Phi$ be a local adjunction, as in Definition 3.1. Each of the linear maps

$$
\Phi_{X, Y}: \mathfrak{K}_{B}\left(X \otimes_{A} F, Y\right) \stackrel{\cong}{\rightarrow} \mathfrak{K}_{A}\left(X, Y \otimes_{B} E\right)
$$

is an isomorphism of operator spaces.

We will see later (Corollary 3.14) that the matrix norms of $\Phi_{X, Y}$ are in fact bounded independently of $X$ and $Y$.

Proof. For a Hilbert $A$-module $X$, we let $X^{\infty}$ denote the orthogonal direct sum of countably many copies of $X$ (see [BLM04, 8.1.9]). For each $n \geq 1$ there is a natural isometric embedding

$$
M_{n}\left(\mathfrak{K}_{A}(X, Z)\right) \longrightarrow \mathfrak{K}_{A}\left(X^{\infty}, Z^{\infty}\right)
$$

that is defined by letting each $n \times n$ matrix act by matrix multiplication on the first $n$ copies of $X$ inside $X^{\infty}$, and by zero on the remaining copies. One also has isometric isomorphisms $\left(X \otimes_{A} F\right)^{\infty} \cong X^{\infty} \otimes_{A} F$. The diagram

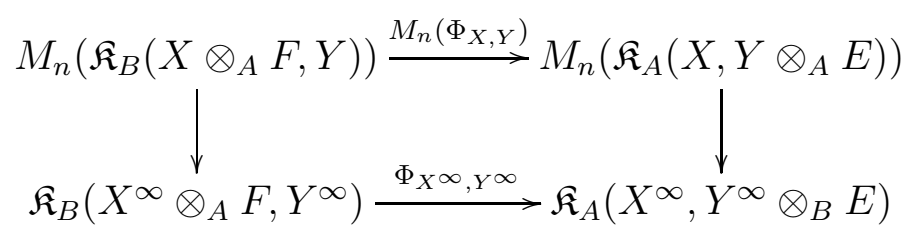

commutes by the naturality of $\Phi$, showing that $\left\|M_{n}\left(\Phi_{X, Y}\right)\right\| \leq\left\|\Phi_{X^{\infty}, Y \infty}\right\|$ for every $n$.

As with adjoints, there is no distinction between left and right local adjunctions: given a local adjunction $\Phi$ as above, we may define a second local adjunction,

$$
\Phi_{X, Y}^{*}: \mathfrak{K}_{A}\left(Y \otimes_{B} E, X\right) \stackrel{\cong}{\longrightarrow} \mathfrak{K}_{A}\left(Y, X \otimes_{A} F\right)
$$


by means of the formula

$$
\Phi_{X, Y}^{*}: T \mapsto\left(\Phi_{X, Y}\right)^{-1}\left(T^{*}\right)^{*} .
$$

This interchanges the roles played by the correspondences $E$ and $F$ in Definition 3.1. And as with adjoints, it is very relevant to study units and counits associated to a local adjunction. The following definition merely repeats Definition 2.20 in the present context.

Definition 3.4. Let $\Phi$ be a local adjunction, as in Definition 3.1 .

(a) A unit for $\Phi$ is a bounded, adjointable $A$-bimodule map

$$
\eta: A \longrightarrow F \otimes_{B} E
$$

that defines $\Phi$ by means of the commuting diagram

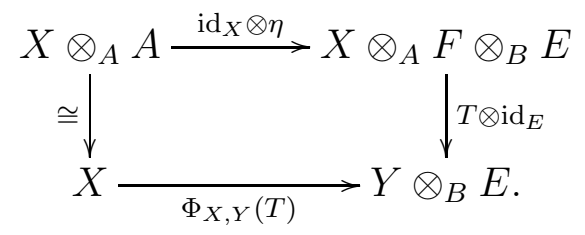

(b) A counit for $\Phi$ is a bounded, adjointable $B$-bimodule map

$$
\varepsilon: E \otimes_{A} F \longrightarrow B
$$

that defines the inverse of the isomorphism $\Phi_{X, Y}$ by means of the commuting diagram

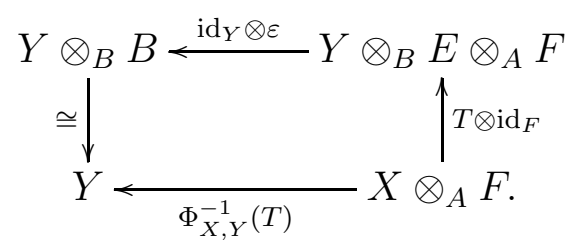

Once again, these definitions are symmetric with respect to the transposition $\Phi \leftrightarrow \Phi^{*}$ given in (3.3) above. If $\eta$ is a unit for $\Phi$, then the adjoint operator $\eta^{*}$ is a counit for $\Phi^{*}$, while if $\varepsilon$ is a counit for $\Phi$, then $\varepsilon^{*}$ is a unit for $\Phi^{*}$. 
Lemma 3.5. A local adjunction admits at most one unit and at most one counit.

Proof. Let $\eta: A \rightarrow F \otimes_{B} E$ be a unit, and let $u_{\lambda}$ be an approximate unit in the $C^{*}$-algebra $\mathfrak{K}_{B}(F, F)$. The map $\eta$ is the strong-operator limit of the net $\left(u_{\lambda} \otimes \operatorname{id}_{E}\right) \circ \eta$, and the unit property of $\eta$ identifies this net with $\Phi_{A, F}\left(u_{\lambda}\right)$. Thus $\eta$ is uniquely determined by $\Phi$. The uniqueness of counits follows by symmetry from the uniqueness of units.

A local adjunction need admit neither a unit nor a counit, or it might admit one without the other. But in the examples of interest to us at least one will exist. It is also the case that every local adjunction admits a bounded (but not necessarily adjointable) counit $\varepsilon: F \otimes_{A} E \rightarrow B$. See Section 3.5.

If a functor has an adjoint, then the adjoint is unique up to a canonical natural isomorphism. Local adjoints are, in general, only unique in the following weaker sense:

Lemma 3.6. Suppose that a correspondence $F$ from $A$ to $B$ is a local adjoint to a correspondence $E$ from $B$ to $A$, and also to a second correspondence $G$ from $B$ to $A$. There is a canonical completely bounded isomorphism $E \cong G$ of $B$-A operator bimodules.

Proof. The two local adjunctions give completely bounded isomorphisms

$$
E \cong \mathfrak{K}_{A}(A, E) \cong \mathfrak{K}_{B}(F, B) \cong \mathfrak{K}_{A}(A, G) \cong G
$$

of $B$ - $A$-bimodules.

Remarks 3.7. The converse of Lemma 3.6 is also true: Theorems 2.37 and 2.38 together imply that up to natural isomorphism, $\mathfrak{K}_{A}\left(X, Y \otimes_{B} E\right)$ depends only on the operator bimodule structure of $E$. In the course of proving Theorem 3.10, below, we will in fact establish a bijection between the set of local adjunctions between $F$ and $E$, and the set of $B$ - $A$ operator bimodule isomorphisms $F^{*} \cong E$.

On the question of uniqueness, we will later see (Proposition 3.34) that if there exists a counit

$$
\varepsilon: E \otimes_{A} F \longrightarrow B,
$$

then the canonical isomorphism $E \cong G$ of Lemma 3.6 is adjointable, and so furnishes a natural isomorphism between the tensor product functors $E$ and 
$G$. In particular, if the tensor product functor $F$ has an adjoint, then it has a unique local adjoint. In the absence of a counit, a tensor product functor may admit several local adjoints that are not isomorphic to one another as correspondences. See Section 4.5.

\subsection{Local Adjunctions from Adjunctions}

Lemma 3.8. If $F: \mathcal{H}_{A} \rightarrow \mathcal{H}_{B}$ is a tensor product functor with an adjoint $E: \mathcal{H}_{B} \rightarrow \mathcal{H}_{A}$, then for all $X \in \mathcal{H}_{A}$ and all $Y \in \mathcal{H}_{B}$, the natural isomorphism

$$
\Phi_{X, Y}: \mathfrak{B}_{B}\left(X \otimes_{A} F, Y\right) \rightarrow \mathfrak{B}_{A}\left(X, Y \otimes_{B} E\right)
$$

maps $\mathfrak{K}_{B}\left(X \otimes_{A} F, Y\right)$ isomorphically onto $\mathfrak{K}_{A}\left(X, Y \otimes_{B} E\right)$.

Proof. The space $\mathfrak{K}_{B}\left(X \otimes_{A} F, Y\right)$ is densely spanned by operators of the form

$$
L=y \otimes(K x \otimes f)^{*},
$$

where $y \in Y, x \in X, f \in F, K \in \mathfrak{K}_{A}(X, X)$, and we are using the notation of (2.4). The naturality of $\Phi$ gives

$$
\Phi_{X, Y}(L)=\Phi_{X, Y}\left(\left(y \otimes(x \otimes f)^{*}\right) \circ\left(K^{*} \otimes 1_{F}\right)\right)=\Phi_{X, Y}\left(y \otimes(x \otimes f)^{*}\right) \circ K^{*},
$$

which is compact because $K^{*}$ is. This shows that $\Phi$ maps compact operators into compact operators, and a similar argument applied to $\Phi^{-1}$ shows that the map is a bijection.

Corollary 3.9. Every adjunction of tensor product functors

$$
\Phi_{X, Y}: \mathfrak{B}_{B}\left(X \otimes_{A} F, Y\right) \stackrel{\cong}{\longrightarrow} \mathfrak{B}_{A}\left(X, Y \otimes_{B} E\right)
$$

restricts to a local adjunction

$$
\Phi_{X, Y}: \mathfrak{K}_{B}\left(X \otimes_{A} F, Y\right) \stackrel{\cong}{\longrightarrow} \mathfrak{K}_{A}\left(X, Y \otimes_{B} E\right) .
$$




\subsection{Characterization of Local Adjunctions}

Theorem 3.10. A tensor product functor from $\mathcal{H}_{A}$ to $\mathcal{H}_{B}$, induced from a correspondence $F$, has a locally adjoint tensor product functor if and only if both of the following conditions are met:

(a) The conjugate $B$-A-bimodule $F^{*}$ carries an A-valued inner product making it into a correspondence from $B$ to $A$.

(b) The conjugate operator space structure on $F^{*}$ is completely boundedly equivalent to the Hilbert A-module operator space structure on $F^{*}$.

Proof. First suppose that $E$ is a local adjoint to $F$. The isomorphism

$$
\Phi_{A, B}: \mathfrak{K}_{B}(F, B) \stackrel{\cong}{\longrightarrow} \mathfrak{K}_{A}(A, E)
$$

gives, using Lemma 2.9, an isomorphism of $B$ - $A$-bimodules

$$
F^{*} \stackrel{\cong}{\longrightarrow} E
$$

that is, in addition, an operator space isomorphism. The $A$-valued inner product on $F^{*}$ inherited from $E$ via this isomorphism satisfies conditions (a) and (b).

Conversely, suppose we are given a compatible $A$-valued inner product making $F^{*}$ into a correspondence from $B$ to $A$. Let us introduce a second symbol, $E$, for this correspondence, and give $E$ the operator space structure it inherits from its Hilbert $A$-module structure (in contrast, we assign to $F^{*}$ the operator space structure it receives as the conjugate of $F$ ). We have sequences of natural isomorphisms of operator spaces

$$
\begin{aligned}
\mathfrak{K}_{B}\left(X \otimes_{A} F, Y\right) & \cong Y \otimes_{h B}\left(X \otimes_{A} F\right)^{*} \\
& \cong Y \otimes_{h B}\left(X \otimes_{h A} F\right)^{*} \\
(2) & \cong Y \otimes_{h B} F^{*} \otimes_{h A} X^{*}
\end{aligned}
$$

and

$$
\begin{aligned}
Y \otimes_{h B} E \otimes_{h A} X^{*} & \cong Y \otimes_{B} E \otimes_{h A} X^{*} \\
& \cong \underset{(5)}{\cong} \mathfrak{K}_{A}\left(X, Y \otimes_{B} E\right),
\end{aligned}
$$


as follows. The isomorphisms (1) and (5) come from Theorem 2.38, while (2) and (4) come from Theorem 2.37. The isomorphism (3) is a result of the compatibility of the Haagerup tensor product with the conjugation operation on operator spaces. If we assume that the identity map from $F^{*}$ to $E$ is a completely bounded isomorphism of operator spaces, then of course

$$
Y \otimes_{h B} F^{*} \otimes_{h A} X^{*} \cong Y \otimes_{h B} E \otimes_{h A} X^{*},
$$

and we can link all of the displayed isomorphisms together to obtain a local adjunction between $F$ and $E$.

Since a local adjunction between $F$ and $E$ determines canonically an operator space isomorphism $E \cong F^{*}$-and conversely - we will usually just write $F^{*}$ instead of $E$ from now on, keeping in mind that this implies the choice of a suitable $A$-valued inner product on $F^{*}$.

Remarks 3.11. The proof gives a very simple formula for the adjunction isomorphism

$$
\Phi_{X, Y}: \mathfrak{K}_{B}\left(X \otimes_{A} F, Y\right) \rightarrow \mathfrak{K}_{A}\left(X, Y \otimes_{B} F^{*}\right)
$$

namely

$$
\Phi_{X, Y}\left(y \otimes(x \otimes f)^{*}\right)=\left(y \otimes f^{*}\right) \otimes x^{*} .
$$

We also note that, given a local adjunction between $F$ and $F^{*}$, the equivalence of the two operator space structures on $F^{*}$ allows us to combine Theorems 2.37 and 2.38 to obtain canonical isomorphisms

$$
\mathfrak{K}_{B}(F, F) \cong F \otimes_{B} F^{*} \quad \text { and } \quad \mathfrak{K}_{A}\left(F^{*}, F^{*}\right) \cong F^{*} \otimes_{A} F
$$

of $A$ - $A$-bimodules and $B$ - $B$-bimodules, respectively.

Corollary 3.14. Let $\Phi$ be a local adjunction, as in Definition 3.1. The matrix norms of the isomorphisms $\Phi_{X, Y}$ are bounded independently of $X$ and $Y$ :

$$
\left\|\Phi_{X, Y}\right\|_{\mathrm{cb}} \leq\left\|\Phi_{A, B}\right\|_{\mathrm{cb}} \leq\left\|\Phi_{A^{\infty}, B^{\infty}}\right\| .
$$

Proof. Using Theorem 2.38 to identify spaces of compact operators with Haagerup tensor products, the map $\Phi_{X, Y}$ is given by

$$
1_{Y} \otimes \Phi_{A, B} \otimes 1_{X^{*}}: Y \otimes_{h B} F^{*} \otimes_{h A} X^{*} \rightarrow Y \otimes_{h B} E \otimes_{h A} X^{*} .
$$

The functoriality of the Haagerup tensor product (see BLM04, Lemma 3.4.5]) then gives $\left\|\Phi_{X, Y}\right\|_{\mathrm{cb}} \leq\left\|\Phi_{A, B}\right\|_{\mathrm{cb}}$. Theorem 3.2 gives the second inequality, $\left\|\Phi_{A, B}\right\|_{\mathrm{cb}} \leq\left\|\Phi_{A^{\infty}, B^{\infty}}\right\|$. 


\subsection{Local Adjunctions and Representations}

In addition to the tensor product functors $F: \mathcal{H}_{A} \rightarrow \mathcal{H}_{B}$ studied in the previous sections, every correspondence $F$ from $A$ to $B$ induces a functor

$$
F=F \otimes_{B}:{ }_{B} \mathcal{H} \longrightarrow{ }_{A} \mathcal{H}
$$

between the categories of (nondegenerate) Hilbert space representations of $A$ and $B$. In this section we shall prove the following result.

Theorem 3.15. Let $F$ be a correspondence from $A$ to $B$, and let $E$ be a correspondence from $B$ to $A$. Every local adjunction between $F$ and $E$ gives rise to a (two-sided) adjunction between the tensor product functors

$$
F:{ }_{B} \mathcal{H} \longrightarrow{ }_{A} \mathcal{H} \text { and } E:{ }_{A} \mathcal{H} \longrightarrow{ }_{B} \mathcal{H}
$$

on categories of Hilbert space representations.

Remark 3.16. Under the equivalences explained in Section 3.7, this theorem corresponds to [KPW04, Theorem 4.4(1)]. Compare also [CH16], where an adjunction theorem is formulated in the context of operator modules.

Before beginning the proof let us collect some preliminary facts.

Lemma 3.17. Let $F$ be a correspondence from $A$ to $B$. The formula

$$
\left(f_{1}^{*}, f_{2}\right) \mapsto\left\langle f_{1}, f_{2}\right\rangle
$$

defines a completely contractive map of operator $B$-B-bimodules

$$
F^{*} \otimes_{h A} F \longrightarrow B \text {. }
$$

Proof. If $\bar{S} \in M_{n}\left(F^{*}\right)$ (we shall avoid writing $S^{*}$ to avoid confusion with the matrix adjoint operation) and if $T \in M_{n}(F)$, then the map

$$
\left\langle \_,\right\rangle_{n}: M_{n}\left(F^{*}\right) \times M_{n}(F) \longrightarrow M_{n}(B)
$$

induced from the inner product map in the statement of the lemma, as in Section 2.7, sends the pair $(\bar{S}, T)$ to the Hilbert module inner product

$$
\left\langle S^{\top}, T\right\rangle \in M_{n}(B)
$$

where $S \in M_{n}(F)$ is image of $\bar{S} \in M_{n}\left(F^{*}\right)$ under the conjugate linear isomorphism $M_{n}\left(F^{*}\right) \rightarrow M_{n}(F)$ and $S^{\top}$ is the transpose matrix. So the lemma follows from the universal property of the Haagerup tensor product (cf. Section 2.7]) and the Cauchy-Schwarz inequality [BLM04, p.297]:

$$
\left\|\left\langle S^{\top}, T\right\rangle\right\|_{M_{n}(B)} \leq\left\|S^{\top}\right\|_{M_{n}(F)}\|T\|_{M_{n}(F)}=\|\bar{S}\|_{M_{n}\left(F^{*}\right)}\|T\|_{M_{n}(F)} .
$$


Suppose next we are given a local adjunction

$$
\Phi_{X, Y}: \mathfrak{K}_{B}\left(X \otimes_{A} F, Y\right) \stackrel{\cong}{\longrightarrow} \mathfrak{K}_{A}\left(X, Y \otimes_{B} E\right) .
$$

Use it to identify $E$ with $F^{*}$ as in the proof of Theorem 3.10, and in this way equip $F^{*}$ with the structure of a Hilbert $B$ - $A$-bimodule.

Lemma 3.18. Let $F$ be a correspondence from $A$ to $B$, and assume that $F^{*}$ has been equipped with a Hilbert A-module structure making $F^{*}$ and $F$ local adjoints. The formulas

$$
\varepsilon: f_{1}^{*} \otimes f_{2} \mapsto\left\langle f_{1}, f_{2}\right\rangle \quad \text { and } \quad \delta: f_{1} \otimes f_{2}^{*} \mapsto\left\langle f_{1}^{*}, f_{2}^{*}\right\rangle
$$

define completely bounded bimodule maps

$$
\varepsilon: F^{*} \otimes_{A} F \rightarrow B \quad \text { and } \quad \delta: F \otimes_{B} F^{*} \rightarrow A .
$$

Proof. The assertion about $\varepsilon$ follows from the previous lemma and Theorem 2.37. The assertion about $\delta$ follows from these results together with Theorem 3.10 .

The completely bounded map $\delta: F \otimes_{B} F^{*} \rightarrow A$ given by Lemma 3.18 induces, by Theorem [2.37, a natural bounded Hilbert space operator

$$
\delta_{X}: F \otimes_{B} F^{*} \otimes_{A} X \longrightarrow X
$$

for each $X \in{ }_{A} \mathcal{H}$. Being a bounded operator between Hilbert spaces, $\delta_{X}$ has an adjoint operator

$$
\eta_{X}: X \longrightarrow F \otimes_{B} F^{*} \otimes_{A} X,
$$

and we obtain a natural transformation from the identity functor on ${ }_{A} \mathcal{H}$ to the tensor product functor

$$
F \circ F^{*}: X \mapsto F \otimes_{B} F_{A}^{*} \otimes X
$$

We need just such a natural transformation in order to prove that the tensor product functor $F^{*}:{ }_{A} \mathcal{H} \rightarrow{ }_{B} \mathcal{H}$ is left adjoint to $F$ (namely the unit of the adjunction). We also need a natural transformation

$$
\varepsilon_{Y}: F^{*} \otimes_{A} F \otimes_{B} Y \longrightarrow Y
$$


for every $Y \in{ }_{B} \mathcal{H}$ (this is the counit of the adjunction), and then we need to show that the compositions

$$
F \otimes_{B} Y \stackrel{\eta_{F(Y)}}{\longrightarrow} F \otimes_{B} F^{*} \otimes_{A} F \otimes_{B} Y \stackrel{F\left(\varepsilon_{Y}\right)}{\longrightarrow} F \otimes_{B} Y
$$

and

$$
F^{*} \otimes_{A} X \stackrel{F^{*}\left(\eta_{X}\right)}{\longrightarrow} F^{*} \otimes_{A} F \otimes_{B} F^{*} \otimes_{A} X \stackrel{\varepsilon_{F^{*}(X)}}{\longrightarrow} F^{*} \otimes_{A} X
$$

are the identity. See [ML98, IV.1 Theorem 2].

We shall define $\varepsilon_{Y}$ to be the map

$$
F^{*} \otimes_{A} F \otimes_{B} Y \stackrel{\varepsilon \otimes \operatorname{id}_{Y}}{\longrightarrow} A \otimes_{A} Y \stackrel{\cong}{\longrightarrow} Y
$$

obtained from the completely bounded inner product map $\varepsilon: F^{*} \otimes_{A} F \rightarrow B$ in Lemma 3.18. Having done so, (3.21) and (3.22) become effectively equivalent: just reverse the roles of $F$ and $F^{*}$ to get from one to the other. So it remains to prove (3.21).

Lemma 3.23. Let $u_{\lambda}$ be an approximate unit for the $C^{*}$-algebra $\mathfrak{K}_{B}(F, F)$, viewed as a net in $F \otimes_{B} F^{*}$ using the isomorphism of Theorem 2.38. The adjoint operator $\eta_{X}=\delta_{X}^{*}$ in (3.20) is given by the formula

$$
\eta_{X}(x)=\lim _{\lambda \rightarrow \infty} u_{\lambda} \otimes x
$$

where the limit exists in the weak topology on the Hilbert space $F \otimes_{B} F^{*} \otimes_{A} X$. Proof. We need to prove that

$$
\lim _{\lambda \rightarrow \infty}\left\langle u_{\lambda} \otimes x, v\right\rangle=\left\langle x, \delta_{X} v\right\rangle
$$

for all $v \in F \otimes_{B} F^{*} \otimes_{A} X$. To this end, let us show that

$$
\langle T \otimes x, v\rangle=\left\langle x, \delta_{X} T^{*} v\right\rangle,
$$

where $T \in \mathfrak{K}_{B}(F, F)$ and where on the right hand side of the identity the adjoint operator $T^{*}$ acts on the triple tensor product $F \otimes_{B} F^{*} \otimes_{A} X$ by acting on the first factor alone. To prove (3.24) it suffices to calculate with elementary tensors

$$
T=f_{1} \otimes f_{2}^{*} \quad \text { and } \quad v=f_{3} \otimes f_{4}^{*} \otimes x_{1},
$$

and this straightforward using the formulas for the Hilbert space inner products given in Definition 2.12. 
Proof of Theorem 3.15. Let us show that for every $Y \in{ }_{B} \mathcal{H}$ the composition (3.21) is equal to the identity map. Let $f \in F, y \in Y$ and $z \in F \otimes_{B} Y$. It suffices to show that

$$
\left\langle\left(F\left(\varepsilon_{Y}\right) \eta_{F(Y)}(f \otimes y), z\right\rangle=\langle f \otimes y, z\rangle .\right.
$$

If we write $w=F\left(\varepsilon_{Y}\right)^{*} z$ then (3.25) becomes the identity

$$
\left\langle\eta_{F(Y)}(f \otimes y), w\right\rangle=\langle f \otimes y, z\rangle .
$$

Using the formula for $\eta_{F(Y)}$ proved in the lemma, the left hand side of (3.26) is

$$
\lim _{\lambda \rightarrow \infty}\left\langle u_{\lambda} \otimes f \otimes y, w\right\rangle,
$$

or in other words

$$
\lim _{\lambda \rightarrow \infty}\left\langle F\left(\varepsilon_{Y}\right)\left(u_{\lambda} \otimes f \otimes y\right), z\right\rangle .
$$

From the definition of $\varepsilon_{Y}$ we have

$$
F\left(\varepsilon_{Y}\right): f_{1} \otimes f_{2}^{*} \otimes f_{3} \otimes y \mapsto f_{1}\left\langle f_{2}, f_{3}\right\rangle \otimes y
$$

and this implies that for every $T \in \mathfrak{K}_{B}(F, F) \cong F \otimes_{B} F^{*}$ one has

$$
F\left(\varepsilon_{Y}\right)(T \otimes f \otimes y)=T f \otimes y .
$$

Applying this to (3.27) we find that

$$
\left\langle\left(F\left(\varepsilon_{Y}\right) \eta_{F(Y)}(f \otimes y), z\right\rangle=\lim _{\lambda \rightarrow \infty}\left\langle u_{\lambda} f \otimes y, z\right\rangle,\right.
$$

and (3.25) follows from this.

The uniqueness of adjoint functors in the usual context of category theory implies:

Corollary 3.28. If $F$ admits local adjoints $E$ and $G$, then the tensor product functors

$$
E \otimes_{A}, G \otimes_{A}:{ }_{A} \mathcal{H} \longrightarrow{ }_{B} \mathcal{H}
$$

are canonically isomorphic. 


\subsection{Existence of Units and Counits}

We continue to work with locally adjoint Hilbert modules $F$ and $F^{*}$ as in Theorem 3.10, but let us return now from Hilbert space representations back to Hilbert modules. For each Hilbert $B$-module $Y$ we have a bounded, $B$ linear map

$$
\operatorname{id}_{Y} \otimes \varepsilon: Y \otimes_{B} F^{*} \otimes_{A} F \rightarrow Y \otimes_{B} B
$$

defined by identifying the Hilbert module tensor products with Haagerup tensor products, and using the functoriality of the latter with respect to completely bounded maps. A short computation using (3.12) shows that for each Hilbert $A$-module $X$, and each $T \in \mathfrak{K}_{A}\left(X, Y \otimes_{B} F^{*}\right)$, the diagram

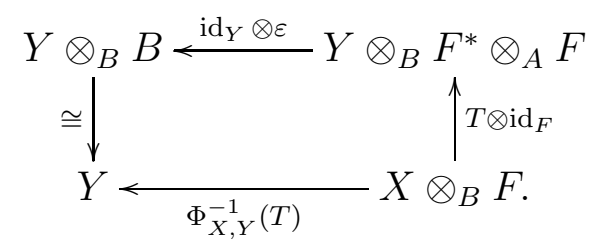

is commutative: thus $\varepsilon$ is almost a counit for the adjunction, its only defect being that it might not be adjointable. The action homomorphism $\eta: A \rightarrow$ $\mathfrak{B}_{B}(F, F)$ may similary be considered a kind of generalised unit, a point of view justified by the following proposition.

Proposition 3.29. The following are equivalent:

(a) There exists a unit $A \rightarrow F \otimes_{B} F^{*}$ for the local adjunction.

(b) The natural isomorphism $\Phi_{X, Y}: \mathfrak{K}_{B}\left(X \otimes_{A} F, Y\right) \rightarrow \mathfrak{K}_{A}\left(X, Y \otimes_{B} F^{*}\right)$ extends to a natural transformation $\mathfrak{B}_{B}\left(X \otimes_{A} F, Y\right) \rightarrow \mathfrak{B}_{A}\left(X, Y \otimes_{B} F^{*}\right)$.

(c) The action of $A$ on $F$ is through a $*$-homomorphism from $A$ into $\mathfrak{K}_{B}(F, F)$.

(d) The map $\delta: F \otimes_{B} F^{*} \rightarrow$ A of Lemma 3.18 is adjointable.

If $A$ is unital, one may add a fifth equivalent condition:

(e) F is finitely generated as a right B-module.

When these conditions hold, the unit in (a) is equal to $\delta^{*}$; the natural transformation in (b) is 1-1; and the *-homomorphism in (c) corresponds to $\eta$ under the canonical identification of $\mathfrak{K}_{B}(F, F)$ with $F \otimes_{B} F^{*}$. 
Proof. That (a) implies (b) is clear. To prove (b) implies (c), let us first show that $\Phi_{X, Y}$ is one-to-one on $\mathfrak{B}_{B}\left(X \otimes_{A} F, Y\right)$. Fix a nonzero $T \in \mathfrak{B}_{B}\left(X \otimes_{A}\right.$ $F, Y)$, and choose $y \in Y$ such that $T^{*} y \neq 0$. Then the compact operator $\left(T^{*} y\right)^{*}=y^{*} \circ T \in \mathfrak{K}_{B}\left(X \otimes_{A} F, B\right)$ is nonzero, so its image under $\Phi_{X, B}$ is nonzero. By naturality we have

$$
\Phi_{X, B}\left(y^{*} \circ T\right)=\left(y^{*} \otimes 1_{F^{*}}\right) \circ \Phi_{X, Y}(T),
$$

and so $\Phi_{X, Y}(T) \neq 0$.

Now let $a$ be an element of $A=\mathfrak{K}_{A}(A, A)$. By naturality, $\Phi_{A, F}\left(a \otimes 1_{F}\right)=$ $a \circ \Phi_{A, F}\left(1_{F}\right) \in \mathfrak{K}_{A}\left(A, F \otimes_{B} F^{*}\right)$. Since $\Phi_{A, F}$ is $1-1$, and maps $\mathfrak{K}_{B}(F, F)$ surjectively onto $\mathfrak{K}_{A}\left(A, F \otimes_{B} F^{*}\right)$, we conclude that $a \otimes 1_{F}$ is compact: i.e., $A$ acts on $F$ by compact operators.

Next, we show that (c) implies (d). Identifying $F \otimes_{B} F^{*}$ with $\mathfrak{K}_{B}(F, F)$ as in (3.13), one finds the following formula for the $A$-valued inner product:

$$
\left\langle K_{1}, K_{2}\right\rangle_{\mathfrak{K}_{B}(F, F)}=\delta\left(K_{1}^{*} K_{2}\right) .
$$

Letting $\eta: A \rightarrow \mathfrak{K}_{B}(F, F)$ be the action homomorphism, we find that

$$
\langle\eta(a), K\rangle_{\mathfrak{K}_{B}(F, F)}=\delta\left(\eta\left(a^{*}\right) K\right)=a^{*} \delta(K)=\langle a, \delta(K)\rangle_{A},
$$

where we have used that $\delta$ is a map of $A$-bimodules. Thus $\delta$ is adjointable, with adjoint $\eta$.

Finally (d) implies (a), as follows. If $\delta$ is adjointable, then Lemma 3.18 implies that $\delta$ is a counit for the local adjunction $\Phi^{*}$, and so $\delta^{*}$ is a unit for $\Phi$.

If $A$ is unital, then the equivalence of (c) and (e) follows from the wellknown fact ([BLM04, 8.1.27]) that the identity operator on a Hilbert $B$ module $F$ is compact if and only if $F$ is finitely generated.

Corollary 3.31. Let $F$ be a correspondence from $A$ to $B$, admitting a local adjoint $F^{*}$ and a unit $\eta: A \rightarrow F \otimes_{B} F^{*}$. The $C^{*}$-algebra $A$ decomposes as the direct sum of two-sided ideals

$$
A=A_{F^{*}} \oplus A_{F^{*}}^{\perp}
$$

where $A_{F^{*}}=\overline{\operatorname{span}}\left\{\left\langle f_{1}^{*}, f_{2}^{*}\right\rangle \mid f_{1}^{*}, f_{2}^{*} \in F^{*}\right\}$ and $A_{F^{*}}^{\perp}=\operatorname{ker}\left(A \rightarrow \mathfrak{B}_{B}(F, F)\right)$. 
Proof. By Proposition 3.29, the existence of a unit $\eta$ implies that $\delta$ is adjointable, with $\delta^{*}=\eta$. Being a homomorphism of $C^{*}$-algebras, $\eta$ has closed range and so [Lan95, Theorem 3.2] gives

$$
A=\text { image } \delta \oplus \operatorname{ker} \eta=A_{F^{*}} \oplus A_{F^{*}}^{\perp}
$$

as (right) Hilbert $A$-modules. Both $\delta$ and $\varepsilon$ are left $A$-linear, so the above is a decomposition of $C^{*}$-algebras.

Recalling the relationship between units and counits, Proposition 3.29 and Corollary 3.31 immediately give:

Proposition 3.32. The following are equivalent:

(a) There exists an (adjointable) counit $F^{*} \otimes_{A} F \rightarrow B$.

(b) The natural isomorphism $\Phi_{X, Y}^{-1}: \mathfrak{K}_{A}\left(X, Y \otimes_{B} F^{*}\right) \rightarrow \mathfrak{K}_{B}\left(X \otimes_{A} F, Y\right)$ extends to a natural transformation $\mathfrak{B}_{A}\left(X, Y \otimes_{B} F^{*}\right) \rightarrow \mathfrak{B}_{B}\left(X \otimes_{A} F, Y\right)$.

(c) The action of $B$ on $F^{*}$ is through a*-homomorphism from $B$ into the $C^{*}$-algebra $\mathfrak{K}_{A}\left(F^{*}, F^{*}\right)$.

(d) The map $\varepsilon: F^{*} \otimes_{A} F \rightarrow B$ of Lemma 3.18 is adjointable.

If $B$ is unital, one may add a fifth equivalent condition:

(e) F is finitely generated as a left A-module.

When these conditions hold, the counit in (a) is equal to $\varepsilon$; natural transformation in (b) is 1-1; the *-homomorphism in (c) corresponds to $\varepsilon^{*}$ under the canonical identification of $\mathfrak{K}_{A}\left(F^{*}, F^{*}\right)$ with $F^{*} \otimes_{A} F$; and the $C^{*}$-algebra $B$ decomposes as the direct sum of two-sided ideals $B=B_{F} \oplus B_{F}^{\perp}$.

Corollary 3.33. A local adjunction extends to an adjunction if and only if it admits both a unit and a counit.

Proof. As we observed above (Proposition 2.21), the "only if" direction is a standard fact about adjoint functors. For the converse, assume that a local adjunction

$$
\Phi_{X, Y}: \mathfrak{K}_{B}\left(X \otimes_{A} F, Y\right) \rightarrow \mathfrak{K}_{A}\left(X, Y \otimes_{B} F^{*}\right)
$$

admits a unit and a counit. Proposition 3.29 gives an extension of $\Phi$ to a $1-1$ natural transformation $\Phi_{X, Y}: \mathfrak{B}_{B}\left(X \otimes_{A} F, Y\right) \rightarrow \mathfrak{B}_{A}\left(X, Y \otimes_{B} F^{*}\right)$, while 
Proposition 3.32 gives a 1-1 natural transformation $\Psi_{X, Y}: \mathfrak{B}_{A}\left(X, Y \otimes F^{*}\right) \rightarrow$ $\mathfrak{B}_{B}\left(X \otimes_{A} F, Y\right)$ such that $\Phi$ and $\Psi$ are mutually inverse on the subspaces of compact operators. For each $T \in \mathfrak{B}_{B}\left(X \otimes_{A} F, Y\right)$ and $y \in Y$ we have

$$
y^{*} \circ \Psi_{X, Y} \Phi_{X, Y}(T)=\Psi_{X, B} \Phi_{X, B}\left(y^{*} \circ T\right)=y^{*} \circ T
$$

in $\mathfrak{K}_{B}\left(X \otimes_{A} F, B\right)$. Since the maps $y^{*}: Y \rightarrow B$ separate the points of $Y$, the above calculation implies that $\Psi_{X, Y} \circ \Phi_{X, Y}=\mathrm{id}$. A similar computation shows that $\Phi_{X, Y} \circ \Psi_{X, Y}=\mathrm{id}$, and so $\Phi$ is a natural isomorphism.

Combining the above results, we recover Theorem 2.24.

\subsection{Uniqueness of Local Adjoints}

Local adjoints are unique up to completely bounded bimodule isomorphism, by Lemma 3.6. They are not, in general, unique as correspondences: see Section 4.5. However, the stronger form of uniqueness does hold in the presence of a counit:

Proposition 3.34. Let $F$ be a correspondence from $A$ to $B$ admitting a local adjoint $E$ and an (adjointable) counit

$$
\varepsilon: E \otimes_{A} F \longrightarrow B
$$

If $G$ is a second local adjoint to $F$, then $E$ and $G$ are isomorphic as correspondences from $B$ to $A$.

The proposition is an easy consequence of the following two lemmas.

Lemma 3.35. Let $E$ and $G$ be Hilbert $A$-modules and let $T: E \rightarrow G$ be a bounded, A-linear operator (not necessarily adjointable). If $K$ is any compact (and hence adjointable) operator on the Hilbert module $E$, and if $g \in G$, then the operator

$$
\begin{gathered}
S_{K, g}: E \longrightarrow A \\
S_{K, g}: e \longmapsto\langle g, T K e\rangle_{G}
\end{gathered}
$$

is adjointable (and indeed compact). 
Proof. Since the compact operators are a norm-closed subspace of all operators, it suffices to prove the lemma in the special case where $K=e_{1} \otimes e_{2}^{*}$. In this case the operator in the lemma is the compact operator

$$
S_{K, g}=\left\langle g, T e_{1}\right\rangle_{G} \otimes e_{2}^{*}: E \longrightarrow A,
$$

and so the proof is complete.

Lemma 3.36. Let $E$ and $G$ be correspondences from $B$ to $A$. If the action of $B$ on $E$ is through compact operators, then every bounded, $A$-B-linear operator $T: E \rightarrow G$ is adjointable.

Proof. We need to show that for every $g \in G$ there is some $f \in E$ such that

$$
\langle g, T e\rangle_{G}=\langle f, e\rangle_{E}
$$

for every $e \in E$. By the factorization theorem (applied twice), the element $g$ may be written as a product $b_{1} g_{1} a_{1}$ with $b_{1} \in B$ and $g_{1} \in G$, and $a_{1} \in A$. Next, if $e \in E$, then

$$
\left\langle b_{1} g_{1}, T e\right\rangle_{G}=\left\langle g_{1}, b_{1}^{*} T e\right\rangle_{G}=\left\langle g_{1}, T b_{1}^{*} e\right\rangle_{G},
$$

where in the last step we are using the $B$-linearity of $T$. Since $b_{1}^{*}$ is acting on $E$ as a compact operator, it follows from the previous lemma that we can write

$$
a^{*}\left\langle g_{1}, T b_{1}^{*} e\right\rangle_{G}=\langle S a, e\rangle_{E}
$$

for some operator $S: A \rightarrow E$ and every $a \in A$. Hence if we set $f=S a_{1}$, then

$$
\langle g, T e\rangle_{G}=\left\langle b_{1} g_{1} a_{1}, T e\right\rangle_{G}=a_{1}^{*}\left\langle g_{1}, T b_{1}^{*} e\right\rangle_{G}=\langle f, e\rangle_{E},
$$

as required.

Proof of Proposition 3.34. This is an immediate consequence of the previous lemma, together with Proposition 3.32, which tells us that $B$ acts on $E$ through compact operators, and Lemma 3.6 , which tells us that $E$ and $G$ are isomorphic as $B$ - $A$-operator bimodules.

Corollary 3.37. If $F$ admits an adjoint $E$, then $E$ is the unique local adjoint to $F$. 


\subsection{Left and Right Indexes}

The purpose of this section is to explicate the relationship between our results and those of [KPW04]. Throughout this section, $F$ denotes a correspondence from $A$ to $B$, and we assume that $F^{*}$ has been equipped with an $A$-valued inner product making it into a correspondence from $B$ to $A$.

Following [KPW04, Definitions 2.8 and 2.9], we say that $F$ is of finite numerical index if there are positive constants $l$ and $r$ such that for all $f_{1}, \ldots, f_{n} \in F$,

$$
\left\|\sum\left\langle f_{i}, f_{i}\right\rangle\right\|_{B} \leq l\left\|\sum f_{i}^{*} \otimes f_{i}\right\|_{\mathfrak{K}_{A}\left(F^{*}, F^{*}\right)}
$$

and

$$
\left\|\sum\left\langle f_{i}^{*}, f_{i}^{*}\right\rangle\right\|_{A} \leq r\left\|\sum f_{i} \otimes f_{i}^{*}\right\|_{\mathfrak{K}_{B}(F, F)} .
$$

The smallest $l$ and $r$ for which these inequalities hold are called, respectively, the left numerical index and the right numerical index of $F$.

Lemma 3.38. The correspondence $F$ is of finite numerical index if and only if $F^{*}$ is a local adjoint to $F$.

Proof. If $F^{*}$ is a local adjoint to $F$ then Lemma 3.18, combined with the identifications $\mathfrak{K}_{B}(F, F) \cong F \otimes_{B} F^{*}$ and $\mathfrak{K}_{A}\left(F^{*}, F^{*}\right) \cong F^{*} \otimes_{A} F$, implies that the map

$$
\varepsilon: \mathfrak{K}_{A}\left(F^{*}, F^{*}\right) \rightarrow B, \quad f_{1}^{*} \otimes f_{2} \mapsto\left\langle f_{1}, f_{2}\right\rangle
$$

is bounded, and this ensures that $F$ has finite left numerical index (equal to $\|\varepsilon\|)$. Reversing the roles of $F$ and $F^{*}$ shows that $F$ also has finite right numerical index, equal to the norm of the map

$$
\delta: \mathfrak{K}_{B}(F, F) \rightarrow A, \quad f_{1} \otimes f_{2}^{*} \mapsto\left\langle f_{1}^{*}, f_{2}^{*}\right\rangle .
$$

To prove the converse, denote by $E$ the bimodule $F^{*}$ equipped with the operator space structure coming from the $A$-valued inner product; $F^{*}$ will denote the same bimodule with its conjugate operator space structure. If $F$ has finite right numerical index $r$, then for $f^{*} \in F^{*}$ one has

$$
\left\|f^{*}\right\|_{E}=\left\|\left\langle f^{*}, f^{*}\right\rangle\right\|_{A}^{1 / 2} \leq r\left\|f \otimes f^{*}\right\|_{\mathfrak{K}_{B}(F, F)}^{1 / 2}=r\|\langle f, f\rangle\|_{B}^{1 / 2}=r\left\|f^{*}\right\|_{F^{*}},
$$

showing that the identity map $E \rightarrow F^{*}$ has norm at most $r$. Similarly, if $F$ has finite left numerical index $l$, then the identity map $F^{*} \rightarrow E$ has norm at 
most $l$. Two successive applications of [KPW04, Corollary 2.10] show that, if $F$ has left numerical index $l$ and right numerical index $r$, then so does $M_{n}(F)$ (viewed as a correspondence from $M_{n}(A)$ to $M_{n}(B)$ ). The above argument then shows that the identity map $M_{n}(E) \rightarrow M_{n}\left(F^{*}\right)$ has norm at most $r$, and its inverse has norm at most $l$. Therefore the identity $E \rightarrow F^{*}$ is completely bounded, and so $E$ is a local adjoint to $F$ by Theorem 3.10 .

Suppose $F$ has finite numerical index. The maps $\varepsilon$ and $\delta$ may be extended to normal maps

$$
\varepsilon^{\prime \prime}: \mathfrak{K}_{A}\left(F^{*}, F^{*}\right)^{\prime \prime} \rightarrow B^{\prime \prime} \quad \text { and } \quad \delta^{\prime \prime}: \mathfrak{K}_{B}(F, F)^{\prime \prime} \rightarrow A^{\prime \prime}
$$

between the enveloping von Neumann algebras. Following KPW04, Definition 2.17], we say that $F$ has finite left index if the image $\varepsilon^{\prime \prime}(1)$ of the identity lies in the multiplier algebra of $B$. The condition of having finite right index is defined analogously, in terms of the map $\delta^{\prime \prime}$. Say $F$ has finite index if it has finite left index and finite right index.

Lemma 3.39. Suppose $F$ has finite numerical index, so that $F^{*}$ is a local adjoint to $F$.

(a) $F$ has finite left index if and only if there is a counit $F^{*} \otimes_{A} F \rightarrow B$ for the local adjunction.

(b) $F$ has finite right index if and only if there is a unit $A \rightarrow F \otimes_{B} F^{*}$ for the local adjunction.

(c) F has finite index if and only if the local adjunction is an adjunction.

Proof. It is shown in [KPW04, Theorem 2.22] that $F$ has finite left (respectively, right) index if and only if $B$ (resp. $A$ ) acts on $F^{*}$ (resp. $F$ ) by compact operators. The asserted equivalences now follow from Propositions 3.29 and 3.32 and from Corollary 3.33

These results are summarised in the following table, which relates our terminology to that of [KPW04]:

\begin{tabular}{|r|l|}
\hline The functor $\otimes_{A} F$ has & if and only if the correspondence $F$ has \\
\hline a local adjoint & finite numerical index \\
a local adjoint with unit & finite numerical index and finite right index \\
a local adjoint with counit & finite numerical index and finite left index \\
an adjoint & finite index \\
\hline
\end{tabular}




\section{Examples}

\subsection{Commutative Examples}

Let $X$ be a compact Hausdorff space. The category of Hilbert modules over the $C^{*}$-algebra $C(X)$ is equivalent to the category of continuous fields of Hilbert spaces over $X$ (and adjointable operators between continuous fields) via the functor that associates to a continuous field its Hilbert module of continuous sections.

A continuous map $\varphi: X \rightarrow Y$ between compact Hausdorff spaces determines a homomorphism of $C^{*}$-algebras from $C(Y)$ to $C(X)$. Let

$$
F:=C(X)
$$

be the associated correspondence from $C(Y)$ to $C(X)$. From the point of view of continuous fields the tensor product functor

$$
\varphi^{*}:=\otimes F: \mathcal{H}_{C(Y)} \rightarrow \mathcal{H}_{C(X)}
$$

associated to $F$ is given by pullback $\varphi^{*}$ of continuous fields of Hilbert spaces along the map $\varphi$.

Coverings. Let us examine the correspondence $F$ above in the case of a surjective map between compact Hausdorff spaces.

Definition 4.1. Let $\pi: X \rightarrow Y$ be a continuous surjection of compact Hausdorff spaces.

(a) The map $\pi$ is a covering if it is an open map, and if $\# \pi^{-1}[y]$ (the number of points in the pre image of $y$ ) is a finite and locally constant function of $y \in Y$.

(b) The map $\pi$ is a branched covering if it is open and if the number $\# \pi^{-1}[y]$ is finite and uniformly bounded over $y \in Y$.

The following is a consequence of results of Pavlov and Troitsky [PT11, combined with Theorem 2.24 and Proposition 4.7 (below):

Proposition 4.2. Let $\pi: X \rightarrow Y$ be a continuous surjection of compact Hausdorff spaces. 
(a) The pullback functor $\pi^{*}$ admits an adjoint if and only if $\pi$ is a covering.

(b) The functor $\pi^{*}$ admits a local adjoint if and only if $\pi$ is a branched covering.

Proof. Theorem 2.24 implies that $\pi^{*}$ admits an adjoint if and only if $F=$ $C(X)$ is finitely generated and projective over $C(Y)$, which by PT11, Theorem 1.3] is equivalent to $\pi$ being a covering. Proposition 4.7 (below) implies that $\pi^{*}$ admits a local adjoint if and only if there is a finite-index conditional expectation $C(X) \rightarrow C(Y)$, which by [PT11, Theorem 1.1] is equivalent to $\pi$ being a branched covering.

The $C(Y)$-valued inner product on the conjugate module $F^{*}=C(X)^{*}$ is given by a formula of the kind

$$
\left\langle f_{1}^{*}, f_{2}^{*}\right\rangle(y)=\sum_{x \in \pi^{-1}[y]} \mu_{y}(x) f_{1}(x) \overline{f_{2}(x)}
$$

where the weight functions $\mu_{y}: \pi^{-1}[y] \rightarrow[0,1]$ are determined (usually not uniquely) by the branching of the cover over the point $y$. For example, if $\pi$ is the quotient map for a finite group action, one can take $\mu_{y}$ to be the constant function $1 / \# \pi^{-1}[y]$ (see Proposition 4.8). For a construction of suitable $\mu_{y}$ for a general branched covering, see [PT11, Proof of Theorem 4.3].

Open Subsets. Now let $X$ be a compact Hausdorff space, and let $U \subset$ $X$ be an open subset. Let $F=C_{0}(U)$. This is a correspondence from $C(X)$ to $C_{0}(U)$, whose associated tensor-product functor $\mathcal{H}_{C(X)} \rightarrow \mathcal{H}_{C_{0}(U)}$ is restriction of continuous fields of Hilbert spaces from $X$ to $U$ (even though $U$ is not compact it is still true that $\mathcal{H}_{C_{0}(U)}$ is equivalent to the category of continuous fields of Hilbert spaces over $U$ ).

Proposition 4.3. The functor of restriction to $U$ has a local adjoint, namely extension of continuous fields by zero, which is represented by $E=C_{0}(U)$ viewed as a correspondence from $C_{0}(U)$ to $C(X)$. The local adjoint is an adjoint if and only if $U$ is both open and closed in $X$.

Proof. This is a special case of Proposition 4.5, below. 
Infinite-Dimensional Fibers. In the above examples, the bimodules that occur, viewed as continuous fields of Hilbert spaces, have finite fiber dimensions, namely fiber dimensions zero or one. Interesting examples with infinite fiber dimension do not occur in the purely commutative context. For instance, if $H$ is a Hilbert space, viewed as a correspondence from $\mathbb{C}$ to $\mathbb{C}$, then $H$ has a local adjoint if and only if $H$ is finite-dimensional (in which case the local adjoint is an adjoint).

\subsection{Relation with Morita Equivalences}

Let $F$ be a correspondence from $A$ to $B$, and suppose that $F^{*}$ has been given an $A$-valued inner product making it into a correspondence from $B$ to $A$, such that

$$
\left\langle x^{*}, y^{*}\right\rangle z=x\langle y, z\rangle
$$

for all $x, y, z \in F$. This condition says that $F$ and $F^{*}$ restrict to mutually inverse (strong) Morita equivalences between the ideals $A_{F^{*}} \subseteq A$ and $B_{F} \subseteq$ $B$ : see [BLM04, 8.1.2].

Proposition 4.5. The correspondences $F$ and $F^{*}$ are local adjoints. They are adjoints if and only if the ideals $A_{F^{*}}$ and $B_{F}$ are complemented.

Proof. The equality (4.4) implies that the two norms on $F^{*}$ are equal: see [BLM04, Lemma 8.1.15]. The equality (4.4) is also satisfied by the induced inner products on $M_{n}(F)$ and $M_{n}\left(F^{*}\right)$, and so the identity map on $F^{*}$ is a complete isometry between the two operator structures; thus $F$ and $F^{*}$ are local adjoints, by Theorem 3.10 .

If $F$ and $F^{*}$ are adjoints, then the ideals $A_{F^{*}}$ and $B_{F}$ are complemented by Corollary 3.31 and Proposition 3.32. Conversely, suppose that $A_{F^{*}}$ is complemented in $A$. The formula (4.4) implies that the elements of $A_{F^{*}}$ act on $F$ by $B$-compact operators. On the other hand, (4.4) implies that $F=\mathfrak{K}_{B}(F, F) F=A_{F^{*}} F$, showing that the complementary ideal $A_{F^{*}}^{\perp}$ acts by zero on $F$. Therefore $A$ acts by compact operators on $F$, and Proposition 3.29 thus implies that the local adjunction admits a unit; a similar argument proves the existence of a counit.

The local adjunctions for which the inner products satisfy (4.4) are precisely those for which the adjunction isomorphisms

$$
\Phi: \mathfrak{K}_{B}\left(X \otimes_{A} F, Y\right) \stackrel{\cong}{\longrightarrow} \mathfrak{K}_{A}\left(X, Y \otimes_{B} F^{*}\right)
$$


are isomorphisms of ternary rings of operators, meaning that

$$
\Phi\left(R S^{*} T\right)=\Phi(R) \Phi(S)^{*} \Phi(T)
$$

for every $R, S, T \in \mathfrak{K}_{B}\left(X \otimes_{A} F, Y\right)$. (This is an immediate consequence of the formula (3.12) for $\Phi$; we owe this observation to an anonymous referee.) This is in turn equivalent to the condition that the isomorphisms $\Phi$ be isometries, or equivalently complete isometries, as follows from a theorem of Hamana, Kirchberg and Ruan [BLM04, Corollary 4.4.6] and a naturality argument as in Theorem 3.2 .

\subsection{Conditional Expectations}

Let $A$ be a $*$-subalgebra of a $C^{*}$-algebra $C$, and assume that $A$ contains an approximate unit for $C$. Recall that a conditional expectation from $C$ to $A$ is a positive, idempotent, $A$ - $A$-bimodule map $\varphi: C \rightarrow A$.

Following Frank and Kirchberg [FK98, Theorem 1], we make the following definition.

Definition 4.6. A conditional expectation $\varphi: C \rightarrow A$ is of finite index if one of the following equivalent conditions is satisfied:

(a) $\varphi\left(c^{*} c\right) \neq 0$ whenever $c \neq 0$, and $C$ is complete in the norm

$$
\|c\|_{\varphi}^{2}=\left\|\varphi\left(c^{*} c\right)\right\|
$$

(b) There is a $\lambda \geq 0$ such that $\lambda \varphi-\operatorname{id}_{C}: C \rightarrow C$ is a positive map.

(c) There is a $\kappa \geq 0$ such that $\kappa \varphi-\mathrm{id}_{C}: C \rightarrow C$ is a completely positive map.

Proposition 4.7. Let $F$ be a correspondence from $A$ to $B$ and suppose that in fact $A \subseteq \mathfrak{K}_{B}(F, F)$. The correspondence $F$ admits a local adjoint if and only if there is a finite-index conditional expectation $\varphi: \mathfrak{K}_{B}(F, F) \rightarrow A$.

Proof. Suppose given a finite-index conditional expectation as in the statement of the proposition. Define an $A$-valued inner product on $F^{*}$ by

$$
\left\langle f_{1}^{*}, f_{2}^{*}\right\rangle=\varphi\left(f_{1} \otimes f_{2}^{*}\right)
$$


Condition (a) in Definition 4.6 ensures that $F^{*}$ is a correspondence from $B$ to $A$. Denote by $E$ this correspondence, with the operator space structure it inherits from the $A$-valued inner product. The canonical map $F^{*} \rightarrow E$ is completely bounded because $\varphi$, like all conditional expectations, is completely bounded. The finite index condition (c) implies that the inverse map $E \rightarrow F^{*}$ is also completely bounded, since for each $f^{*}=\left[f_{i, j}^{*}\right] \in M_{n}\left(F^{*}\right)$ we have the estimate

$$
\begin{aligned}
\kappa\left\|f^{*}\right\|_{M_{n}(E)}^{2}=\left\|M_{n}(\kappa \varphi)\left(f \otimes_{M_{n}(B)} f^{*}\right)\right\|_{M_{n}(A)} & \geq\left\|f \otimes_{M_{n}(B)} f^{*}\right\|_{M_{n}\left(\mathfrak{K}_{B}(F, F)\right)} \\
& =\left\|f^{*}\right\|_{M_{n}\left(F^{*}\right)}^{2} .
\end{aligned}
$$

Thus Theorem 3.10 implies that $F$ and $E$ are local adjoints.

Conversely, suppose that $F$ has a local adjoint, and again denote by $E$ the conjugate space $F^{*}$ with the operator space structure it inherits from the given $A$-valued inner product. The $A$-valued inner product determines a map of operator spaces

$$
F \otimes_{B} F^{*} \longrightarrow A
$$

thanks to Lemma 3.18, and so thanks to Theorem 2.38, a (completely) positive map

$$
\psi: \mathfrak{K}_{B}(F, F) \longrightarrow A
$$

of $A$ - $A$-bimodules that sends the elementary compact operator $f_{1} \otimes f_{2}^{*}$ on $F$ to $\left\langle f_{1}^{*}, f_{2}^{*}\right\rangle_{A}$.

Now let $T=\sum_{i=1}^{n} x_{i} \otimes y_{i}^{*} \in \mathfrak{K}(F)$. We compute that

$$
\psi\left(T^{*} T\right)=\sum_{i, j=1}^{n}\left\langle\left\langle x_{i}, x_{j}\right\rangle y_{j}^{*}, y_{i}^{*}\right\rangle_{A}
$$

where the inside inner product is the $B$-valued inner product of $F$, and hence, after a further computation, that $\left\|\psi\left(T^{*} T\right)\right\|$ is the norm of the element

$$
\left(\begin{array}{ccc}
\left\langle x_{1}, x_{1}\right\rangle & \ldots & \left\langle x_{1}, x_{n}\right\rangle \\
\vdots & & \vdots \\
\left\langle x_{n}, x_{1}\right\rangle & \ldots & \left\langle x_{n}, x_{n}\right\rangle
\end{array}\right)^{\frac{1}{2}}\left(\begin{array}{c}
y_{1}^{*} \\
\vdots \\
y_{n}^{*}
\end{array}\right) \in M_{n}(E)
$$

On the other hand a third computation shows that the $M_{n}\left(F^{*}\right)$-norm of this element is $\left\|T^{*} T\right\|$. Using the fact that the norms on $E$ and $F^{*}$ are completely equivalent we find that

$$
\left\|\psi\left(T^{*} T\right)\right\| \geq \text { constant } \cdot\left\|T^{*} T\right\|
$$


for some constant independent of $T$.

The restriction of the map $\psi$ to $A \subseteq \mathfrak{K}_{B}(F, F)$ is multiplication by some positive and central element of the multiplier algebra of $A$. The computation above shows that this element is invertible. Adjusting $\psi$ by multiplying with the inverse of this element we obtain a finite-index conditional expectation, as required.

Here is an example that we shall use when analyzing parabolic induction in Section 5. Let $W$ be a finite group acting by *-automorphisms on a $C^{*}$-algebra $B$, and also acting projectively by twisted automorphisms on a Hilbert $B$-module $F$. This means that associated to each $w \in W$ there is a $\mathbb{C}$-linear operator

$$
U_{w}: F \longrightarrow F
$$

such that

(i) $U_{w}(f b)=U_{w}(f) w(b)$ for all $f \in F$, all $b \in B$, and

(ii) $\left\langle U_{w}\left(f_{1}\right), U_{w}\left(f_{2}\right)\right\rangle=w\left(\left\langle f_{1}, f_{2}\right\rangle\right)$ for all $f_{1}, f_{2} \in F$.

and such that moreover

(iii) $U_{w_{1}}\left(U_{w_{2}}(f)\right)=U_{w_{1} w_{2}}(f) u\left(w_{1}, w_{2}\right)$

for some unitary $u\left(w_{1}, w_{2}\right)$ in the multiplier algebra of $B$. The formula

$$
w(T)=U_{w} T U_{w}^{-1}: F \longrightarrow F
$$

defines a genuine action of $W$ by automorphisms on the $C^{*}$-algebra $\mathfrak{K}_{B}(F, F)$.

Proposition 4.8. Let $W$ be a finite group acting projectively by twisted automorphisms on a Hilbert $B$-module $F$, as above, and let $A$ be the fixed-point algebra $\mathfrak{K}_{B}(F, F)^{W}$. Then $F$, considered as a correspondence from $A$ to $B$, admits a unique local adjoint $E$ for which the canonical action of $W$ is isometric. The isomorphisms

$$
\Phi_{X, Y}: \mathfrak{K}_{B}\left(X \otimes_{A} F, Y\right) \rightarrow \mathfrak{K}_{A}\left(X, Y \otimes_{B} E\right)
$$

for this local adjunction satisfy

$$
\left\|\Phi_{X, Y}\right\|_{\mathrm{cb}}=1 \quad \text { and } \quad\left\|\Phi_{X, Y}^{-1}\right\|_{\mathrm{cb}} \leq|W|^{1 / 2} .
$$


Remark 4.9. The canonical action on $E$ referred to in the statement of the proposition is the one coming from the identification of $E$ with $F^{*}$ that the local junction implies. And the term isometric refers to the given Banach space structure on $E$.

Proof of the Proposition. The conditional expectation

$$
\varphi: \mathfrak{K}_{B}(F, F) \rightarrow A, \quad \varphi(T):=\frac{1}{|W|} \sum_{w \in W} w(T)
$$

has finite index, since if $T \geq 0$ then

$$
|W| \varphi(T)-T=\sum_{w \neq 1} w(T) \geq 0 .
$$

Therefore the correspondence $F$ admits a local adjoint, namely the $B$ - $A$ bimodule $F^{*}$, with $A$-valued inner product

$$
\left\langle f_{1}^{*}, f_{2}^{*}\right\rangle=\varphi\left(f_{1} f_{2}^{*}\right)
$$

The action of $W$ on $E$ is isometric, because $\varphi$ is $W$-invariant.

Suppose $G$ is another local adjoint to $F$, and let $\psi: \mathfrak{K}_{B}(F, F) \rightarrow A$ denote the finite-index conditional expectation induced by the inner product on $G$ as in Proposition 4.7. The action of $W$ on $G$ is isometric if and only if $\psi$ is $W$-invariant, which is to say, if and only if $\psi=\varphi$. Thus $E$ is unique.

For the bounds on the norms, recall from Corollary 3.14 that

$$
\left\|\Phi_{X, Y}\right\|_{\mathrm{cb}} \leq\left\|\Phi_{A, B}\right\|_{\mathrm{cb}}
$$

The same argument shows that $\left\|\Phi_{X, Y}^{-1}\right\|_{\mathrm{cb}} \leq\left\|\Phi_{A, B}^{-1}\right\|_{\mathrm{cb}}$. The conditional expectation $\varphi$ is completely bounded, with cb-norm equal to 1 , and this implies that $\Phi_{A, B}$ has cb-norm 1 . The map $|W| \varphi-\mathrm{id}: B \rightarrow B$ is completely positive, and this implies that

$$
\left\|\Phi_{A, B}^{-1}\right\|_{\mathrm{cb}} \leq|W|^{1 / 2}
$$

as required.

\subsection{Direct Sums}

Direct sums of local adjoints are not, in general, local adjoints: for example, $\mathbb{C}$ is an adjoint correspondence from $\mathbb{C}$ to $\mathbb{C}$, but the countable direct sum 
$\mathbb{C}^{\infty} \cong \ell^{2}(\mathbb{N})$ does not possess a local adjoint as correspondence from $\mathbb{C}$ to $\mathbb{C}$. We may however consider $\mathbb{C}^{\infty}$ as a correspondence on the $C^{*}$-algebraic direct sum $\bigoplus^{\infty} \mathbb{C} \cong C_{0}(\mathbb{N})$, and viewed in this way $\mathbb{C}^{\infty}$ does have a local adjoint, as we shall see.

Suppose we are given two $C^{*}$-algebra direct sums

$$
A=\bigoplus_{\alpha} A_{\alpha} \quad \text { and } \quad B=\bigoplus_{\beta} B_{\beta} .
$$

Suppose we are given a map $\beta \mapsto \iota(\beta)$ from the index set for the decomposition of $B$ to the index set for the decomposition of $A$. For each index $\beta$ let $F_{\beta}$ be a correspondence from $A_{\iota(\beta)}$ to $B_{\beta}$. The external direct sum is

$$
F=\bigoplus_{\beta} F_{\beta}=\left\{\left(f_{\beta}\right) \in \prod F_{\beta}:\left\|f_{\beta}\right\| \rightarrow 0 \text { as } \beta \rightarrow \infty\right\},
$$

with the obvious $A$ - $B$-bimodule structure and $B$-valued inner product.

We shall assume throughout the rest of this section that the map $\iota$ on indices is finite-to-one; indeed we shall assume that it is uniformly finite-toone, in the sense that

$$
\sup _{\beta} \# \iota^{-1}[\beta]<\infty .
$$

Proposition 4.11. Let $F_{\beta}$ be a family of correspondences from $A_{\iota(\beta)}$ to $B_{\beta}$, as above, and assume that condition (4.10) holds. Suppose that each $F_{\beta}$ has a local adjoint $E_{\beta}$, such that the isomorphisms $\varphi_{\beta}: F_{\beta}^{*} \rightarrow E_{\beta}$ satisfy

$$
\sup _{\beta}\left\{\left\|\varphi_{\beta}\right\|_{\mathrm{cb}},\left\|\varphi_{\beta}^{-1}\right\|_{\mathrm{cb}}\right\}<\infty .
$$

Then $F$ has a local adjoint.

Proof. Let

$$
E=\bigoplus_{\beta} E_{\beta}=\left\{\left(e_{\beta}\right) \in \prod E_{\beta}:\left\|e_{\beta}\right\| \rightarrow 0 \text { as } \beta \rightarrow \infty\right\},
$$

This is a correspondence from $A$ to $B$, and the map

$$
\varphi=\bigoplus_{\beta} \varphi_{\beta}: \bigoplus_{\beta} F_{\beta}^{*} \rightarrow \bigoplus E_{\beta}
$$


is an isomorphism of $B$ - $A$-bimodules. Moreover

$$
F^{*}=\bigoplus_{\beta} F_{\beta}^{*}
$$

and so $\varphi$ is also an isomorphism of operator spaces.

\subsection{Miscellany}

\subsubsection{Units and Counits}

Let $B$ be an ideal in $A$, and consider $F=B$ as a correspondence from $A$ to $A$, with the obvious bimodule structure and the inner product $\left\langle b_{1}, b_{2}\right\rangle=b_{1}^{*} b_{2}$. Then $F$ is locally adjoint to itself, but the local adjunction admits neither a unit nor a counit unless $B$ is complemented in $A$. Replacing one of the two acting copies of $A$ by $B$ gives an example of a local adjunction with a unit but no counit, or vice versa.

\subsubsection{Distinct Local Adjoints}

Let $B=C[-1,1]$, and let $A=C[0,1]$, considered as a subalgebra of $B$ via the surjective map $x \mapsto|x|,[-1,1] \rightarrow[0,1]$. Let $F=B$, considered as a correspondence from $A$ to $B$. Define finite-index conditional expectations $\varphi, \psi: B \rightarrow A$ by

$$
\varphi(b)(y):=\frac{1}{2} b(y)+\frac{1}{2} b(-y) \quad \text { and } \quad \psi(b)(y):=\frac{2}{3} b(y)+\frac{1}{3} b(-y) .
$$

The associated correspondences $E=B_{\varphi}$ and $G=B_{\psi}$ from $B$ to $A$ are both locally adjoint to $F$, but $E$ and $G$ are not isomorphic as Hilbert modules. Indeed, if they were isomorphic, then by polar decomposition we could find a unitary isomorphism of bimodules $U: E \rightarrow G$. Unitarity means that $\varphi(b)=\psi\left(b U(1)^{*} U(1)\right)$ for every $b \in B$, but this equality implies that the function $U(1)^{*} U(1)$ is discontinuous at the origin.

\subsubsection{Action by Compact Operators}

Let $A_{i}=B_{i}=\mathbb{C}$ for $i=1,2,3, \ldots$. Let $F_{i}=\mathbb{C}^{i}$, with its canonical Hilbert space structure, and let $E_{i}=\left(\mathbb{C}^{i}\right)^{*}$, with its canonical Hilbert space structure. Considered as a correspondence from $\mathbb{C}$ to $\mathbb{C}$, each $F_{i}$ has $E_{i}$ as its adjoint 
(cf. Example 2.25). The direct sum $F=\bigoplus F_{i}$ does not, however, admit a local adjoint. Indeed, the universal property of direct sums implies that the only possibility for a local adjoint would be the direct sum $E=\bigoplus E_{i}$, but $\bigoplus F_{i}^{*}$ and $\bigoplus E_{i}$ are not isomorphic as operator bimodules over $\bigoplus_{i} \mathbb{C}^{i}$ (cf. Example 2.35). Note that $A=\bigoplus A_{i}$ and $B=\bigoplus B_{i}$ act as compact operators on $F$ and $E$ respectively, showing that condition (b) in Theorem 2.24 is independent of the other conditions.

\subsubsection{Simple $\mathrm{C}^{*}$-Algebras}

Finally, here is a short remark concerning simple $C^{*}$-algebras.

Proposition 4.12. Let $\Phi$ be a local adjunction as in Definition 3.1.

(a) If $A$ is simple, $\Phi$ admits a unit.

(b) If $B$ is simple, $\Phi$ admits a counit.

(c) If $A$ and $B$ are simple, $\Phi$ extends to an adjunction.

Proof. Item (a) follows from Proposition 3.29 and [KPW04, Corollary 2.26]. Item (b) follows by symmetry from (a). Item (c) follows from (a) and (b) by Corollary 3.33 .

\section{Parabolic induction}

In this section we shall analyze the correspondence associated in Cla13 and CCH16 to parabolic induction of tempered unitary group representations.

We shall work with a fixed real reductive group $G$ and standard parabolic subgroup $P$ with Levi factor $L$ and unipotent radical $N$ (thus $P=L N$ is a semidirect product, with $L$ acting on $N$ ). We shall be following the precise conventions of [CCH16, Section 3], but for instance we might take $G$ to be $G L(n, \mathbb{R})$, and $P$ to be a block upper triangular subgroup of $G L(n, \mathbb{R})$, decomposed as a semidirect product of the block upper diagonal matrices acting on the unipotent block upper triangular matrices (with identity matrix blocks down the diagonal).

The groups $G$ and $L$ act on the homogenous space $G / N$ on the left and right, respectively. The vector space $C_{c}^{\infty}(G / N)$ carries left and right actions 
of $G$ and $L$, respectively 11 and it may be completed to a Hilbert $C_{r}^{*}(L)$-module $C_{r}^{*}(G / N)$ on which $C_{r}^{*}(G)$ acts on the left through bounded adjointable operators. See [Cla13, Section 2] or [CCH16, Section 4]. It is this correspondence, from $C_{r}^{*}(G)$ to $C_{r}^{*}(L)$, that we shall study in this section.

The importance of the correspondence $C_{r}^{*}(G / N)$ is that the associated tensor product functor from Hilbert space representations of $C_{r}^{*}(L)$ to $C_{r}^{*}(G)$ may be identified with the functor of parabolic induction from tempered unitary representations of $L$ to tempered unitary representations of $G$. See [Cla13, Section 3]. In [CCH16, Definition 8.2] we defined a correspondence $C_{r}^{*}(N \backslash G)$ from $C_{r}^{*}(L)$ to $C_{r}^{*}(G)$ (the construction is outlined below). Here we shall prove the following result.

Theorem 5.1. The correspondences $C_{r}^{*}(G / N)$ and $C_{r}^{*}(N \backslash G)$ are local adjoints.

Proof. The $C^{*}$-algebras $A=C_{r}^{*}(G)$ and $B=C_{r}^{*}(L)$ both decompose into direct sums

$$
A=\bigoplus_{\alpha} A_{\alpha} \quad \text { and } \quad B=\bigoplus_{\beta} B_{\beta}
$$

The index sets are "associate classes" of pairs consisting of a parabolic subgroup in $G$ or $L$, respectively, and a discrete series representation of the compactly generated part of the parabolic. See CCH16, Proposition 5.16 and Theorem 6.8].

There is a finite-to-one map $\iota$ from the index set for $B$ to the index set for $A$ which comes from the enlargement of standard parabolic subgroups of $L$ to standard parabolic subgroups of $G$ (for instance in the case of $G L(n, \mathbb{R})$, a parabolic subgroup of $L$ consists of a block diagonal group with each block itself a block upper triangular group, and this may be naturally extended to a block upper triangular subgroup of $G L(n, \mathbb{R}))$. The enlargement process preserves Levi factors. The map $\iota$ of associate classes is finite-to-one because non-associate pairs for $B$ can become associate when enlarged to become pairs for $A$. There is a uniform bound on the size of the point inverse images of $\iota$.

The correspondence $F=C_{r}^{*}(G / N)$ from $A$ to $B$ decomposes into an

\footnotetext{
${ }^{1}$ In the case of the $L$-action, there is an adjustment to the standard action that reflects the fact that we should identify $C_{c}(G / N)$ with the space of compactly supported halfdensities on $G / N$.
} 
orthogonal direct sum

$$
F=\bigoplus_{\beta} F_{\beta}
$$

with the same index set as $B$. The inner products of elements in $F_{\beta}$ lie within the summand $B_{\beta} \subseteq B$. The action of $A$ on $F_{\beta}$ factors through the projection onto the summand $A_{\iota(\beta)} \subseteq A$.

The structure of $F_{\beta}$ is a little complicated to recall here (see CCH16, Section 7]). But what is important for us is the structure of the $C^{*}$-algebra $\mathfrak{K}_{B}\left(F_{\beta}, F_{\beta}\right)$, which is given by a formula of the type

$$
\mathfrak{K}_{B}\left(F_{\beta}, F_{\beta}\right) \cong \mathfrak{K}_{C_{0}\left(Z_{\beta}\right)}\left(\mathcal{M}_{\beta}, \mathcal{M}_{\beta}\right)^{W_{\beta}(L)},
$$

where, to use notation that has been streamlined from [CCH16,

(a) $\mathcal{M}_{\beta}$ is a continuous field of parabolically induced tempered unitary representations of $G$ over a locally compact space $Z_{\beta}$ (or in other words a correspondence from $C_{r}^{*}(G)$ to $\left.C_{0}\left(Z_{\beta}\right)\right)$.

(b) $W_{\beta}(L)$ is finite group acting on $Z_{\beta}$ and also acting projectively by twisted automorphisms on $\mathcal{M}_{\beta}$.

The action of $A=C_{r}^{*}(G)$ on $F_{\beta}$ is through the inclusion of the summand $A_{\iota(\beta)}$ as a fixed point algebra

$$
A_{\iota(\beta)}=\mathfrak{K}_{C_{0}\left(Z_{\beta}\right)}\left(\mathcal{M}_{\beta}, \mathcal{M}_{\beta}\right)^{W_{\beta}(G)},
$$

where $W_{\beta}(G)$ is a finite group containing $W_{\beta}(L)$ as a subgroup that also acts on $Z_{\beta}$, and on $\mathcal{M}_{\beta}$ projectively, by twisted automorphisms.

It follows from Proposition 4.8 that $F_{\beta}$ has a local adjoint $F_{\beta}^{*}$, whose $A_{\iota(\beta)^{-}}$ valued inner product is given by averaging over $W_{\beta}(G)$. The finite groups $W_{\beta}(G)$ are uniformly bounded in size, and so it follows from Proposition 4.11 that $F=\oplus_{\beta} F_{\beta}$ has a local adjoint too, namely the direct sum $\oplus_{\beta} F_{\beta}^{*}$. The correspondence $C_{r}^{*}(N \backslash G)$ was defined in [CCH16] to be precisely this direct sum.

We obtain from Theorem 3.15 the following consequence:

Theorem 5.2. The functor of parabolic induction, from tempered unitary Hilbert space representations of $L$ to tempered unitary representations of $G$, possesses a two-sided adjoint. 
Let us note for comparison that, while there is a $C^{*}$-correspondence $C^{*}(G / N)$ implementing parabolic induction for full group $C^{*}$-algebras (see [Cla13]), there is no analogue of Theorem [5.1] in this setting:

Proposition 5.3. Let $G=\mathrm{SL}(2, \mathbb{R})$, and let $P=L N$ be the parabolic subgroup of upper-triangular matrices. The $C^{*}$-correspondence $C^{*}(G / N)$, from $C^{*}(G)$ to $C^{*}(L)$, does not possess a local adjoint.

Proof. Suppose that $C^{*}(G / N)$ does have a local adjoint. Since $L$ is amenable we in fact have $C^{*}(G / N)=C_{r}^{*}(G / N)$, and the left action of $C^{*}(G)$ factors through the quotient mapping $C^{*}(G) \rightarrow C_{r}^{*}(G)$. Now $C_{r}^{*}(G)$ acts on $C_{r}^{*}(G / N)$ by compact operators (see [CCH16, Proposition 4.4]), and so Proposition 3.29 and Corollary 3.31 imply that the kernel $J$ of the action map $C^{*}(G) \rightarrow \mathfrak{B}_{C^{*}(L)}\left(C^{*}(G / N), C^{*}(G / N)\right)$ is a direct summand in $C^{*}(G)$. The closed subset of the unitary dual $\widehat{G}$ corresponding to the ideal $J$ is precisely the principal series, i.e. the set of irreducible constituents of parabolically induced representations of $G$. The principal series is not open in $\widehat{G}$, as there is a complementary series of representations whose closure intersects the princial series (see e.g. [Mil71]). Therefore $J$ is not a direct summand of $C^{*}(G)$, and $C^{*}(G / N)$ has no local adjoint.

We conclude with a remark. The determination of the structure of the correspondence $C_{r}^{*}(G / N)$ relies very heavily on the classification results on the tempered representations due to Harish-Chandra, Langlands and others. It is an interesting open problem to approach the construction of a locally adjoint correspondence from a more geometric starting point, without relying so heavily on representation theory.

\section{References}

[Bee82] W. Beer. On Morita equivalence of nuclear $C^{*}$-algebras. J. Pure Appl. Algebra, 26(3):249-267, 1982.

[Ber87] J. Bernstein. Second adjointness for representations of reductive $p$-adic groups. Draft: http://www.math.uchicago.edu/ mitya/langlands.html, 1987.

[Ble97] D. P. Blecher. A new approach to Hilbert $C^{*}$-modules. Math. Ann., 307(2):253-290, 1997. 
[BLM04] D. P. Blecher and C. Le Merdy. Operator algebras and their modules - an operator space approach, volume 30 of London Mathematical Society Monographs. New Series. The Clarendon Press, Oxford University Press, Oxford, 2004. Oxford Science Publications.

[CCH16] P. Clare, T. Crisp, and N. Higson. Parabolic induction and restriction via $C^{*}$-algebras and Hilbert $C^{*}$-modules. Compositio Mathematica, FirstView:1-33, 22016.

[CH16] T. Crisp and N. Higson. Parabolic induction, categories of representations and operator spaces. In to appear in Operator algebras and their applications: a tribute to Richard $V$. Kadison, volume 671 of Contemp. Math. Amer. Math. Soc., Providence, RI, 2016.

[Cla13] P. Clare. Hilbert modules associated to parabolically induced representations. J. Operator Theory, 69(2):483-509, 2013.

[ER00] E. G. Effros and Z-J. Ruan. Operator spaces, volume 23 of London Mathematical Society Monographs. New Series. The Clarendon Press, Oxford University Press, New York, 2000.

[FK98] M. Frank and E. Kirchberg. On conditional expectations of finite index. J. Operator Theory, 40(1):87-111, 1998.

[GLR85] P. Ghez, R. Lima, and J. E. Roberts. $W^{*}$-categories. Pacific J. Math., 120(1):79-109, 1985.

[KPW04] T. Kajiwara, C. Pinzari, and Y. Watatani. Jones index theory for Hilbert $C^{*}$-bimodules and its equivalence with conjugation theory. J. Funct. Anal., 215(1):1-49, 2004.

[Lan95] E. C. Lance. Hilbert $C^{*}$-modules, volume 210 of London Mathematical Society Lecture Note Series. Cambridge University Press, Cambridge, 1995. A toolkit for operator algebraists.

[Mil71] D. Miličić. Topological representation of the group $C^{*}$-algebra of SL(2, R). Glasnik Mat. Ser. III, 6(26):231-246, 1971.

[ML98] S. Mac Lane. Categories for the working mathematician, volume 5 of Graduate Texts in Mathematics. Springer-Verlag, New York, second edition, 1998. 
[Mor65] K. Morita. Adjoint pairs of functors and Frobenius extensions. Sci. Rep. Tokyo Kyoiku Daigaku Sect. A, 9:40-71 (1965), 1965.

[PT11] A. A. Pavlov and E. V. Troitskii. Quantization of branched coverings. Russ. J. Math. Phys., 18(3):338-352, 2011.

[Ren10] D. Renard. Représentations des groupes réductifs p-adiques, volume 17 of Cours Spécialisés [Specialized Courses]. Société Mathématique de France, Paris, 2010. 